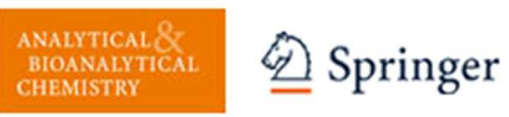

\title{
Exploring matrix effects in liquid chromatography-tandem mass spectrometry determination of pesticide residues in tropical fruits
}

\begin{tabular}{|c|c|}
\hline Journal: & Analytical and Bioanalytical Chemistry \\
\hline Manuscript ID: & $A B C-02214-2014 . R 1$ \\
\hline Type of Paper: & Research Paper \\
\hline Date Submitted by the Author: & 26-Jan-2015 \\
\hline Complete List of Authors: & $\begin{array}{l}\text { Botero-Coy, Ana Maria; University Jaume I, Research Institute for } \\
\text { Pesticides and Water } \\
\text { Marín, Jose; Research Institute of Pesticides and Water, } \\
\text { Sancho, Juan Vicente; University Jaume I, Research Institute for Pesticides } \\
\text { and Water; University Jaume I, Research Institute for Pesticides and Water } \\
\text { Serrano, Roque; University Jaume I, Research Institute for Pesticides and } \\
\text { Water } \\
\text { Hernández, Félix; University Jaume I, Research Institute for Pesticides and } \\
\text { Water }\end{array}$ \\
\hline Keywords: & $\begin{array}{l}\text { Pesticide residue analysis, Tropical fruit, Matrix effect, LC-tandem MS, } \\
\text { Colombia fruit matrices }\end{array}$ \\
\hline
\end{tabular}


Analytical and Bioanalytical Chemistry

Editor in Chief

Castellon, 26th January 2015

Dear Dr. Wise,

Please find enclosed our paper entitled Exploring matrix effects in liquid chromatography-tandem mass spectrometry determination of pesticide residues in tropical fruits, by A.M. Botero-Coy, J.M. Marín, R. Serrano, J.V. Sancho, F. Hernández, which we submit to Analytical and Bioanalytical Chemistry after revision following the referees' comments.

We have answered all the referees' comments (see separate document), and also made a revision of the English trying to simplify the text and to improve the readability of the manuscript.

We hope after this revision the paper can be considered acceptable for publication in Analytical and Bioanalytical Chemistry.

Yours sincerely

Professor Félix Hernández

Research Institute for Pesticides and Water

University Jaume I, Castellon, Spain

felix.hernandez@uji.es 
1

2

3

4

5

6

7

8

9

10

11

12

13

14

15

16

17

18

19

20

21

22

23

24

25

26

27

28

29

30

31

32

33

34

35

36

37

38

39

40

41

42

43

44

45

46

47

48

49

50

51

52

53

54

55

56

57

58

59

60
1 Exploring matrix effects in liquid chromatography-tandem mass spectrometry

2 determination of pesticide residues in tropical fruits

3

4

5

6 Research Institute for Pesticides and Water, University Jaume I, 12071 Castellón,

7

8

9

10

11

$$
\text { Spain }
$$

Tel 34-964-387366; Fax 34-964-387368; felix.hernandez@uij.es 

appreciated characteristics, particularly their high nutritional value and distinctive taste, different to traditional fruits. Due to their introduction in international markets it is necessary to have a reliable analytical methodology available for the sensitive determination of pesticide residues in order to monitor the compliance of maximum residue limits (MRLs)From an analytical point of view, tropical fruits have generally been far less studied than other fruits frequently consumed in the European Union or USA, which are among the most important markets. In this work, LC-MS/MS-based methodology using triple quadrupole analyzer has been developed for the multi-residue determination of selected pesticides and metabolites in tropical fruits, which were selected among the most popular in Colombia, one of the most important suppliers of tropical fruits around the world. After selection of a QuEChERS (Quick, Easy, Cheap, Effective, Rugged and Safe)-based sample treatment, the study was focused on matrix effects evaluation, in order to find a simple way for their correction. Twelve different food matrices were selected to perform this study: the seven Colombian tropical fruits of highest value for domestic and international markets (uchuva, tamarillo, granadilla, gulupa, maracuya, papaya and pithaya), and five more matrices highly consumed in Colombia (lulo, carambolo, feijoa, mangostan and guayaba). Twenty compounds, including pesticides widely applied in tropical fruits pest control and several metabolites considered in residue definition, were used as model compounds in this work. Correction factors were used on the basis of calibration graphs obtained with standards in solvent and in matrix, and their usefulness was supported by validation of the method in all the matrices tested at $0.01 \mathrm{mg} / \mathrm{kg}$ and 0.1 $\mathrm{mg} / \mathrm{kg}$. The analysis of real-world samples revealed the presence of several target compounds that were identified by the acquisition of two MS/MS transitions, and by ion intensity ratio and retention time agreement.

\section{KEYWORDS}

Pesticide residue analysis; tropical fruits; matrix effects, LC-tandem MS, Colombia fruit matrices 


\section{INTRODUCTION}

Tropical fruits are of great importance for the economy of several countries around the world, particularly in South America, Asia or Africa, where agricultural activities are mainly based on these types of crops. They are grown under special climatic conditions that give them particular nutritional and organoleptic characteristics. The demand for tropical fruits has increased in the last years because of their particular characteristics of taste, flavor and vitamin content (e.g Vitamin C), carotenes and antioxidant components [1]. Consequently, there is interest in developing and/or adapting analytical methodologies for the determination of pesticide residues in tropical fruits, in order to monitor the compliance of Maximum Residue Limits (MRL). Moreover, in many of these products, MRLs are set by default at a specific low value (i.e. the limit of determination of an analytical method developed for each pesticide in another (similar) food matrix) [2]. This is due to the lack of studies on residue trials performed in compliance with the principles of Good Laboratory Practices (GLP) directed towards registration of the product and the establishing of MRL. It seems clear that analytical methodologies are currently required for tropical fruits, in order to monitor the compliance of MRLs, but also to facilitate the performance of the analytical part of GLP studies to set-up new MRLs on the basis of new residue trails.

Colombia is one of the main suppliers of exotic fruits in the world. Among the main fruits exported are uchuva, tamarillo, tamarindo, granadilla, pithaya, gulupa and baby banana. The main destinations of these products are The Netherlands, Germany, France, Belgium and Spain. It is worth noting that Colombia is the world's first producer of uchuva . In 2012, the total export value of Colombian uchuva was USD 29.2 million, and it was the most important fruit in International trademark, followed by gulupa (USD 12 million), granadilla (USD 2.9 million), pithaya (USD 2 million), "tomate de árbol" or tamarillo (USD 1.3 million) and, to a lesser extent, maracuya and feijoa, giving a total of USD 48,6 million. During the first term of 2014 , an increase of $14.5 \%$ was observed in tropical fruits exportation in relation to 2013 [3].

The use of Multi-Residue Methods (MRMs) is currently required in the field of Pesticide Residue Analysis (PRA) as the only realistic way to monitor a large number of pesticides in the great number of samples that are commonly analyzed in specialized laboratories. Most MRMs reported for fruits and vegetables in the last decade are based on the use of liquid chromatography coupled to tandem mass spectrometry (LC$\mathrm{MS} / \mathrm{MS}$ ), which is considered the technique of choice for the majority of pesticides and metabolites. Its excellent sensitivity, selectivity and robustness, and its suitability for most pesticides currently used, of medium-high polarity and medium-low volatility, are 
among the main reasons for its wide use [4-14]. However, little attention has been paid to tropical fruits, and there is a general lack of analytical methodology available for these types of food matrices. Some of the methods reported are based on gas chromatography with conventional detectors, and on LC-UVIVIS or fluorescence detectors [15-17], which required the confirmation of positive findings by MS. Papaya, mango and guava are among the most studied tropical matrices [15,16] [18-21].

The results on pesticide residues in a wide monitoring of fruit and vegetable samples from South America revealed the post-harvest fungicides thiabendazole and imazalil, and the insecticide chlorpyrifos as the pesticides most frequently detected [22]. Pesticides detected in tropical fruits, like papaya, mango and passion fruit, were chlorothalonil, dimethoate, thiacloprid, imidacloprid, methomyl, cypermetrin, lamdacyhalotrin, propamocarb, and dithiocarbamates. Recently, a GC-MS multi-residue method based on the use of QuEChERS CEN (European Committee for Standardization) procedure has been developed for 50 pesticides in tropical fruits, and validated for tomato, tamarillo and goldenberries (uchuva). The method was applied to the analysis of samples collected from Antioquia (Colombia), and allowed an initial risk assessment, especially for tomatoes, where several pesticides such carbaryl, carbofuran, diazinon, dimethoate, endosulfan alpha, endosulfan beta and p,p'DDT were detected [23].

Taylor [24] considered matrix effects as the "Achilles heel" of LC-MS based methods. Previously to his paper and especially in the last decade, many articles have been reported dealing with matrix effects in LC-MS/MS methods for pesticide residues in environmental, biological and food matrices [6] [13] [25-35]. Different alternatives are normally applied to remove, minimize and/or correct this undesirable effect [26] [27]. The most popular are the use of matrix-matched standards calibration, the application of clean-up steps along the sample treatment, and the use of appropriate internal standards (commonly, isotope-labeled internal standards ILIS) [6] [26] [35] [36]. In theory, one of the most accurate approaches is standard additions, but unfortunately it increases the number of injections and requires to roughly knowing the analyte concentration in the sample to adjust the additions at the correct level. Moreover, ILIS are expensive and not always commercially available. Their use is rather frequent in single methods for specific pesticides, but not in MRMs where a high number of ILIS would be required. Other possibilities, such as optimization of chromatographic separation and/or MS measurements [26] [37] are less applicable in MRMs involving large numbers of compounds. It has been also reported the selection of a few representative matrices to prepare matrix-matched standards for all type of samples 
116 analyzed, assuming that matrix effects are comparable between similar matrices [38].

117 Thus, Kmellar et al. classified the samples analyzed into three groups for preparation of 118 calibration curves: tomato, representing commodities of high water content; pear for 119 commodities of high sugar content; and orange for those of high acidic content [9]. 120 Dilution of sample extracts can be also used to minimize matrix effects and to make 121 different sample extracts more similar if the method has sufficient sensitivity, a fact that 122 is being more common with the instrumentation available nowadays [32].

123 Matrix effects can lead to both ionization suppression and enhancement. This 124 fact clearly affects quantification of analytes if not properly corrected.But matrix effects 125 may also affect the identification of the compound detected, as this process is normally 126 based on the acquisition of two SRM transitions (in tandem MS methods): one for 127 quantification and the other for confirmation of the identity. Typically, the second 128 transition is less intense than the first one due to the lower abundance of the product 129 ion selected. Thus, strong ionization suppression may hamper the presence of the 130 peak at the second transition, avoiding the confirmation of the compound at low 131 concentrations. In addition, the presence of co-eluting matrix interferences sharing the 132 ions used for quantification and/or confirmation may also affect the ion intensity ratio, 133 hindering its compliance within the tolerances admitted [39] [40]. As a consequence, 134 matrix effects need to be properly corrected; this being one of the most challenging 135 tasks in LC-MS/MS based MRMs.

136 Different sample treatments have been developed for pesticide residue analysis 137 in fruits and vegetables. Among them, the QuEChERS procedure has become the 138 most popular, as illustrated by the high number of references from the first publication 139 [41]. The original procedure was based on extraction with acetonitrile, separation of 140 water from acetonitrile by addition of anhydrous $\mathrm{MgSO}_{4}$ and $\mathrm{NaCl}$, and subsequent 141 clean-up using dispersive solid-phase extraction (d-SPE) with a primary secondary 142 amine (PSA), which efficiently removes many polar interfering substances present in 143 the matrix. From the original unbuffered version published in 2003 [41], different 144 versions/modifications have been reported to improve its applicability to more and more 145 pesticides, especially for $\mathrm{pH}$-dependent pesticides, and more complex sample matrices 146 [42-44]. The most popular accepted versions are the AOAC (Association of Official 147 Agricultural Chemists) Official Method 2007, which uses acetate buffer [45], and the 148 European Committee for Standarization (CEN) Standard Method EN 15662, which 149 uses citrate buffering [46]. A combination of different sorbents can be used in d-SPE to 150 improve the clean-up step. Thus, a mixture of three sorbents (C18, PSA and 151 graphitized carbon black (GCB)) has been shown efficient for most analytes tested 
152 [43]. Recently, a comparison of QuEChERS official methodologies has been made for the multi-residue determination of 33 pesticides in Colombian fruits by GC-MS using large volume injection [47]. The CEN method was preferred since acceptable recoveries were achieved for all analytes. The use of GCB in the clean-up step did not improve the results and it was found not to be much useful for clean-up purposes.

In this article, we have developed an analytical methodology for the LC-MS/MS residue determination of 20 compounds (including 6 metabolites) frequently applied for pest control in tropical fruits. Twelve tropical food matrices were selected among those of highest commercial value in Colombia. Their common and scientific names, taxonomic classification and inclusion in the EU group products for MRLs compliance [2] are included in Figure 1. 9 out of 12 products are included in fresh fruits group (miscellaneous fruits), while the remaining 3 belong to the solanaceae family and are included in vegetable fresh group (fruiting vegetables). Most of MRLs applied to the pesticides and food products studied in this work are set-up at default values of 0.01 , 0.02 or $0.05 \mathrm{mg} / \mathrm{kg}$, which correspond to the limit of determination/quantification of the analytical method (marked as $\left(^{*}\right)$ in Table 1, Supplementary Information). QUEChERS (CEN citrate version) was selected for sample extraction and clean-up, and LC-MS/MS with triple quadrupole was used for analysis.. Special attention was paid to matrix effects, trying to find a simple and generic solution for appropriate correction. The applicability of the method was tested by analyzing samples collected from local markets at Colombia and samples exported to Spain.

\section{Experimental}

\subsection{Reagents and chemicals}

Pesticide reference standards were purchased from Dr. Ehrenstorfer (Augsburg, Germany). HPLC-grade methanol, HPLC-grade acetonitrile (ACN) and acetone for residue analysis, Magnesium sulfate, Sodium Chloride, Sodium hydrogencitrate sesquihydrate and Sodium Citrate were purchased from Scharlau (Barcelona, Spain). HPLC-grade water was obtained by purifying demineralized water in a Milli-Q Gradient A10 (Millipore, Bedford, MA, USA). Formic acid ( $\mathrm{HCOOH}, 98$ - 100\%) and ammonium acetate $\left(\mathrm{NH}_{4} \mathrm{Ac}\right.$, reagent grade) were supplied by Scharlau.

Stock standard solutions were prepared dissolving $50 \mathrm{mg}$, accurately weighted, in $100 \mathrm{~mL}$ of acetone obtaining a final concentration of around $500 \mathrm{mg} / \mathrm{L}$. For LC-MS analysis, the stock solutions were diluted with acetonitrile to prepare individual 
187

188

189

190

191

192

193

194

195

196

197

198

199

200

201

202

203

204

205

206

207

208

209

210

211

212

213

214

215

216

217

218

219

220

221

solutions of around $50 \mathrm{mg} / \mathrm{L}$. From these, mixed solutions of 5 pesticides were prepared by diluting with acetonitrile to obtain a final concentration of $5 \mathrm{mg} / \mathrm{L}$. Working mixed solutions of all pesticides were prepared from the $5 \mathrm{mg} / \mathrm{L}$ solutions by dilution with acetonitrile.

Mixed solutions of $1 \mathrm{mg} / \mathrm{L}$ and $0.1 \mathrm{mg} / \mathrm{L}$ in acetonitrile were used for sample fortification in recovery experiments.

In the clean-up step, two types of 2-mL microcentrifuge tubes for QuEChERS dSPE were used, containing: $150 \mathrm{mg}$ anhydrous $\mathrm{MgSO}_{4}, 25 \mathrm{mg}$ PSA and $25 \mathrm{mg} \mathrm{C} 18$ (XE-29508); or $150 \mathrm{mg}$ anhydrous $\mathrm{MgSO}_{4}$ and $50 \mathrm{mg}$ PSA (XE-29511) (Teknokroma, Barcelona, Spain).

\subsection{Liquid chromatography/Mass Spectrometry}

A Waters Alliance 2795 LC system (Waters, Milford, MA, USA) was interfaced to a Quattro micro triple quadrupole mass spectrometer (Waters) using an orthogonal Zspray-electrospray interface. The LC separation was performed using Atlantis $\mathrm{dC}_{18}$ column $(5 \mu \mathrm{m}, 2.1 \times 100 \mathrm{~mm}$; Waters $)$ at a flow rate of $0.3 \mathrm{~mL} / \mathrm{min}$. The mobile phase used was water/ methanol (both $0.1 \mathrm{mM} \mathrm{NH}_{4} \mathrm{Ac}$ and $0.01 \%(2 \mathrm{mM}) \mathrm{HCOOH}$ ) gradient, where the percentage of methanol changed as follows: $0 \mathrm{~min}, 5 \% ; 1 \mathrm{~min}, 5 \% ; 10 \mathrm{~min}$, $90 \%$; $13 \mathrm{~min}, 90 \%, 14.1 \mathrm{~min}, 5 \%$.

Drying gas as well as nebulising gas was nitrogen (Praxair, Valencia, Spain). The desolvation gas and cone gas flows were adjusted to 600 and $60 \mathrm{~L} / \mathrm{h}$, respectively. Infusion experiments were performed using the built-in syringe pump, directly connected to the interface. For operation in MS/MS mode, the collision gas was argon (99.995\%; Praxair, Valencia, Spain) at a pressure of $2 \times 10^{-3} \mathrm{mbar}$ in the collision cell. Capillary voltage of $3.5 \mathrm{KV}$ in positive mode was used.

The interface temperature was set to $350^{\circ} \mathrm{C}$ and the source temperature to 120 ${ }^{\circ} \mathrm{C}$. Dwell times of $0.1 \mathrm{~s}$ were chosen. Two solvent delays were selected to give an additional clean-up using the built-in divert valve controlled by the Masslynx v.4.1 software, the first one from 0 to $4.5 \mathrm{~min}$ and the second one from 15 to $17 \mathrm{~min}$. The application manager TargetLynx was used to process the quantitative data obtained from calibration standards and from samples.

\subsection{Samples}

Samples used in this study were exported from Colombia to the European Union, specifically to Spain. They were acquired in Spanish markets and hypermarkets from 
222 Barcelona and from Castellon. Then, they were transported to the laboratory and

223

224

225

226

227

228

229

230

231

232

233

234

235

236

237

238

239

240

241

242

243

244

245

246

247

248

249

250

251

252

253

254

255

256 processed for analysis. All samples (commonly 6 individual pieces) were homogenised (pulp, small stones and peel). Stones were removed before triturating only in the case of mangostan, due to their larger size. In the case of uchuva, the calyx was also removed. This group of samples, acquired at Spain, were used for analysis and also as "blank" samples for validation of the method. Another group of samples were collected directly in Bogotá, where they were acquired in a local market. They were processed as indicated above and the triturated sample was stored in the freezer at $<-18^{\circ} \mathrm{C}$. Later, they were transported to Spain were they arrived within a maximum period of time of $24 \mathrm{~h}$. This second group of samples was used for analysis and, also to prepare quality controls (QCs) of the analytical procedure.

\subsection{Recommended procedure}

$10 \mathrm{~g}$ of homogenized sample were accurately weighed (precision $0.1 \mathrm{mg}$ ) in a 50 $\mathrm{mL}$ polypropylene centrifuge tube. Extraction was carried out using $10 \mathrm{~mL}$ acetonitrile, shaking by hand for $1 \mathrm{~min}$. Then, $4 \mathrm{~g}$ Magnesium Sulfate, $1 \mathrm{~g}$ Sodium Chloride, $0.5 \mathrm{~g}$ Sodium Hydrogencitrate Sesquihydrate and $1 \mathrm{~g}$ Sodium Citrate were added and immediately shaken vigorously by hand to prevent formation of $\mathrm{MgSO}_{4}$ agglomerates. The tube was centrifuged at $4600 \mathrm{rpm}$ for $10 \mathrm{~min}$.

For the cleanup step, $1 \mathrm{~mL}$ of the upper ACN extract was poured into a d-SPE tube containing $150 \mathrm{mg} \mathrm{MgSO}$, $25 \mathrm{mg}$ PSA and $25 \mathrm{mg} \mathrm{C18}$. The tubes were shaken on a vortex for $30 \mathrm{~s}$ and centrifuged at $12000 \mathrm{rpm}$ for $7 \mathrm{~min}$. Then, $10 \mu \mathrm{L}$ of the final ACN extract was directly injected into the LC system under the experimental conditions indicated in section 3.1. Quantification of samples was made by external calibration with standards in solvent by applying the correction factors obtained in this work (see section 3.3. matrix effects).

The scheme of the procedure applied in shown in Figure $1 \mathrm{SI}$.

\subsection{Matrix effects evaluation}

For evaluation of matrix effects, matrix-matched calibration was prepared for each matrix type by taking $450 \mu \mathrm{L}$ of the blank sample extract and adding $50 \mu \mathrm{L}$ of the corresponding standard in acetonitrile (between 25 and $5000 \mathrm{ng} / \mathrm{mL}$ ), resulting in final concentrations between 2.5 and $500 \mathrm{ng} / \mathrm{mL}$ ).

Matrix effect was evaluated by calculating the percentage of signal suppression or enhancement using equation: 
257

258

259

260

261

262

263

264

265

266

267

268

269

270

271

272

273

274

275

276

277

278

279

280

281

282

283

284

285

286

287

288

289

290
Slopes difference $=\frac{\text { Matrix Calibration Slope }- \text { Direct Calibration Slope }}{\text { Direct Calibration Slope }} \times 100$ [1]

Then, correction factors were estimated for each sample matrix by using the following equation (for details see Figure $2 \mathrm{SI}$ ):

$$
F=\frac{1}{1+\frac{\text { Slopes difference }(\%)}{100}}
$$

[2]

In analysis of samples, the concentration of the pesticide residue was obtained by multiplying the concentration obtained after application of direct calibration with standards in solvent by the corresponding correction factor (see section 3.3. matrix effects).

\subsection{Validation study}

Fortification of samples for recovery experiments was performed by delivering 1 $\mathrm{mL}$ of $0.1 \mathrm{mg} / \mathrm{L}$ or $1 \mathrm{mg} / \mathrm{L}$ standard mixture solutions in acetonitrile to $10 \mathrm{~g}$ homogenized blank sample in order to yield fortification levels of $0.01 \mathrm{mg} / \mathrm{kg}$ or 0.1 $\mathrm{mg} / \mathrm{kg}$, respectively. The fortified samples were left to stand for $1 \mathrm{~h}$ prior to extraction.

Validation of the method was based on European Union SANCO (DirectorateGeneral for Health and Consumer Protection) guideline [39]. Precision (repeatability, in terms of \% RSD) and accuracy (percentage recoveries) were estimated by recovery experiments at two fortification levels, 0.01 and $0.1 \mathrm{mg} / \mathrm{kg}$ (analyzed in quintuplicate). The limit of quantification (LOQ) objective was set as the lowest concentration that was validated in fortified samples with satisfactory precision (RSD $\leq 20 \%$ ) and recovery (between 70-120\%).

The specificity of the method was evaluated using the quantitative transition (Q) by analysing a procedure blank, a processed blank sample, and a processed blank sample spiked at the LOQ level. The acceptance criteria was that both, procedure and sample blanks, did not present any relevant chromatographic peak at the transition selected $(<30 \%)$.

The limit of detection (LOD), defined as the lowest analyte concentration that could be detected and differentiated from the sample blank, i.e. corresponding to a 
signal-to-noise ratio of 3 , was estimated from the chromatograms of sample extracts fortified at the lowest level tested (i.e. $10 \mathrm{ng} / \mathrm{mL}$ ).

Confirmation of the identity of the compound in samples was carried out by acquisition of two MS/MS transitions and the compliance of the $q / Q$ ratio between samples and reference standards with maximum tolerances of $\pm 30 \%$. The agreement in retention time was also required, with maximum deviation of $\pm 0.2 \mathrm{~min}$ [39].

\section{RESULTS AND DISCUSSION}

In a previous work, we developed analytical methodology for around 30 pesticides in seven tropical fruit matrices selected among the most important for the export market of Colombia: uchuva, maracuya, pithaya, tamarillo, gulupa, papaya and granadilla [38]. The LOQ objective at that work was $0.05 \mathrm{mg} / \mathrm{kg}$, which was satisfactory for most pesticide/matrix combinations in terms of MRLs compliance. However, in a few cases the default MRL values are established in the present regulation at values lower than $0.05 \mathrm{mg} / \mathrm{kg}$, commonly 0.01 and $0.02 \mathrm{mg} / \mathrm{kg}$ [2]. Also, metabolites are included in the residue definition for some pesticides. Therefore, the objective of the present work was to make a selection of pesticides commonly applied in Colombia and to update the analytical methodology in a higher number of tropical fruit matrices. The present work was focused only on those compounds from the previous list [38] that include metabolites in their residue definition (dimethoate, that includes its metabolite omethoate; thiamethoxam/clothianidin; carbofuran/3-hydroxy carbofuran; diuron/3,4dichloroaniline; malathion/malaoxon; parathion methyl/paraoxon methyl). Two pesticides (benomyl and thiodicarb), that are applied in the field as precursors of carbendazim and methomyl respectively, were not considered in this work because of the unlikely presence of these compounds in the samples due to their conversion after application in the field and/or degradation along laboratory sample treatment to carbendazim and methomyl. Those compounds with MRLs default values for tropical fruits matrices below $0.05 \mathrm{mg} / \mathrm{kg}$ were also included in this work (dimethoate; picloram; carbofuran; clomazone; parathion methyl; malathion). Another three compounds were also added in relation to the previous work (imazalil, thiacloprid, thiabendazol) as they have been found in some tropical fruits [16] [22]. Altogether, 14 pesticides and 6 metabolites were selected to perform the present study (Table 1).

\subsection{MS and chromatographic conditions}


All spectra were obtained by infusion of $2.5 \mathrm{mg} / \mathrm{L}$ standard solutions in methanol/water $(50: 50, \mathrm{v} / \mathrm{v})$ at a flow rate of $10 \mu \mathrm{L} / \mathrm{min}$. The highest sensitivity was observed for all compounds in positive ESI. The full-scan spectrum showed the most abundant ions for each compound, which typically corresponded to the protonated molecule. Different cone voltages, between 5 and $50 \mathrm{~V}$, were tested to optimize the abundance of the $[\mathrm{M}+\mathrm{H}]^{+}$ion, selecting the values shown in Table 1.

The formation of sodium adducts (e,g., dimethoate, omethoate, picloram, paraoxon methyl, parathion methyl, malaoxon, 3,4 dicloroaniline, diuron, clomazone and chlorpyrifos), which are poorly fragmented and not much recommendable in MS/MS-based methods, was minimized by adding formic acid and/or ammonium acetate, favoring in this way the formation of the protonated molecule $[\mathrm{M}+\mathrm{H}]^{+}$, finally selected for all precursor ions.

MS/MS experiments were performed at different collision energies. Working under selected reaction monitoring (SRM) mode, the most sensitive transition ( $Q$ ) was used for quantification purposes, while the second one was used for confirmation of the identity (q) (Table 1).

An analytical column Atlantis $\mathrm{dC}_{18}(5 \mu \mathrm{m}, 2.1 \times 100 \mathrm{~mm})$ was selected in this study following the good results obtained in the previous work [38]. In order to optimize the chromatographic conditions, a mixed standard solution with all pesticides at $50 \mathrm{ng} / \mathrm{mL}$ was used. First of all, $\mathrm{MeOH}$ and $\mathrm{ACN}$ were checked as organic solvents in the mobile phase. As the studied compounds were optimized in ESI positive mode, the presence of a protic solvent such as $\mathrm{MeOH}$ improved the sensitivity for all the compounds (with the exception of 3,4-dichloroaniline). Furthermore, the analytes' peak shapes were mostly better with $\mathrm{MeOH}$ than using $\mathrm{ACN}$.

Due to the presence of omethoate, a rather polar compound, the initial percentage of organic phase (methanol) was fixed at $5 \%$ for better retention in the $\mathrm{C}_{18}$ chromatographic column employed. Although the extract injected into the LC-MS/MS $(10 \mu \mathrm{L})$ containing $100 \%$ of organic solvent (acetonitrile), the peak shapes were acceptable. Just in the case of thiabendazole and carbendazim, band broadening was observed. The addition of mobile phase modifiers $\left(\mathrm{HCOOH}\right.$ and $\mathrm{NH}_{4} \mathrm{Ac}$ both in water and $\mathrm{MeOH}$ ) improved peak shape and sensitivity for most of the studied compounds according to the MS infusion experiments carried out in the previous step. Thus, several percentages of ammonium acetate (0.05-1 mM) and formic acid (0.005-0.1\%) were tested both in the aqueous and organic phases. The use of $0.1 \mathrm{mM}$ of $\mathrm{NH}_{4} \mathrm{Ac}$ and 
$0.01 \%$ of formic acid was selected as a compromise between satisfactory peak shape and sensitivity for all compounds,.

Finally, the chromatographic conditions selected were: an Atlantis $\mathrm{dC}_{18}$ column with $\mathrm{MeOH}: \mathrm{H}_{2} \mathrm{O}$ (both $0.1 \mathrm{mM} \mathrm{NH}_{4} \mathrm{Ac}$ and $0.01 \% \mathrm{HCOOH}$ ) as mobile phase at a flow rate of $0.3 \mathrm{~mL} / \mathrm{min}$, with a gradient where the percentage of $\mathrm{MeOH}$ changed as follows: $0 \mathrm{~min}, 5 \%$; $1 \mathrm{~min}, 5 \%$; $10 \mathrm{~min}, 90 \%$; $13 \mathrm{~min}, 90 \%$; $14.1 \mathrm{~min}, 5 \%$. Under these conditions, the compounds eluted as shown in Table 1, with retention times between $5.7 \mathrm{~min}$ (omethoate) and $13.3 \mathrm{~min}$ (chlorpyrifos). In order to achieve satisfactory number of points per chromatographic peak (at least 10), the two SRM transitions per compound were distributed in individual functions. Under the final conditions selected, matrixmatched standards at $50 \mathrm{ng} / \mathrm{mL}$ were also injected to test the chromatographic behavior of the analytes in the matrices tested. A similar behavior was observed in all tropical fruits in relation to retention times and peak shape.

373

\subsection{Sample treatment}

In this work, we applied the QuEChERS citrate-buffering version [46]. The d-SPE clean-up was made with a mixture of $\mathrm{MgSO}_{4}, \mathrm{PSA}$ and $\mathrm{C}_{18}$. A scheme of the procedure applied is shown in Figure 1 SI.

After application of the extraction step, two different clean-up systems were tested: a mixture of $\mathrm{MgSO}_{4}, \mathrm{PSA}$ and $\mathrm{C}_{18}$ by one side, and $\mathrm{MgSO}_{4}$ and PSA by other side. The addition of $\mathrm{C}_{18}$ together with the primary-secondary amine (PSA) in the dSPE step has been reported to improve the cleanup for some samples, particularly those that contain lipids such as olives, and it does no harm in any case [44]. Although some chemists employ a freeze-out step to reduce lipid coextractives, $C_{18}$ in d-SPE is faster and easier, and has been shown to work equally well in removing lipids, although freezing out also precipitates additional matrix components having limited solubility in QuEChERS extracts [48].

Not significant differences were found in recoveries and matrix effects among the two clean-up methods tested, although slightly better results were found for the mixture $\mathrm{MgSO}_{4}, \mathrm{PSA}$ and $\mathrm{C}_{18}$. Therefore, this was the approach used in this work. The results obtained for picloram were not satisfactory, as it could not be properly recovered after the QuEChERS procedure applied. Surely, the retention of this acidic analyte (pKa 2.3) in PSA material was the main reason of the low recoveries. This is in agreement with the literature, as low recoveries for this compound have been reported in food matrices [49] [50]. Degradation of picloram by amino or PSA sorbents has been also suggested 
as the reason of the low recoveries consistently obtained when these columns are used with spiked extracts [50]. The low recoveries for acidic compounds when using PSA for clean-up has been widely reported [51].

\subsection{Matrix effects}

As stated in the Introduction, matrix effects are one of the main problems associated to LC-MS/(MS) methods. Among the different possibilities to minimize and/or compensate this undesirable effect, the most popular in MRMs is the use of matrix-matched standards calibration. It is also common to select a few representative matrices to prepare matrix-matched standards when performing routine analysis of large number of samples, assuming that matrix effects are comparable i between similar matrices [9] [29] [33] [34] [38] [44] [49].

In this work, a detailed study of matrix effects was made by comparison of standards prepared in solvent and in matrix, a common way to test matrix effects. The comparison of slopes obtained from calibration curves constructed in the presence of matrix and in pure solvent has been also used to evaluate signal suppression or enhancement [9] [29] [44] [52]. Both, ionization suppression and enhancement were observed depending on the analyte/matrix combination under study. As an alternative to the use of matrix-matched standards calibration for every matrix analyzed, we tested a simple way that avoids the preparation of matrix-matched standards every time that a set of samples needs to be analyzed. The approach consisted on preparing the calibration curves for every analyte in solvent and in the twelve tropical matrices studied to evaluate whether ionization suppression or enhancement took place from the slopes of the calibration graphs. The differences in slopes between calibration in solvent and in matrix were calculated according to equation [1], and the correction factors were estimated for every analyte in every matrix using equation [2]. These correction factors can be applied in future analysis, allowing performing analysis without the need of preparing new calibrations in matrix, just using standards in solvent.

As illustrative example, Figure 2 shows the differences in calibration graphs for several compounds investigated. From this figure, it is easy to appreciate the enhancement ionization for methomyl in several matrices, as, lulo, mangostan and granadilla (Figure $2 \mathrm{a}$ ), the absence of matrix effects for dimethoate (only slight enhancement observed for lulo) (Figure 2b), and the matrix suppression occurring in several matrices for thiacloprid (Figure 2c) and in most matrices for chlorpyrifos (Figure $2 d)$. 
The correction factors resulting from this experiment are summarized in Figure 3.

432

433

434

435

436

437

438

439

440

441

442

443

444

445

446

447

448

449

450

451

452

453

454

455

456

457

458

459

460

461

462

463

464

465
We assumed that no relevant matrix effect occurred when differences in slopes between calibration in matrix and in solvent were up to $\pm 20 \%$. Therefore, no correction was applied in those cases (uncolored boxes). Green/dark boxes refer to matrix enhancement effects (slope difference above 20\%), and yellow/light boxes refer to matrix suppression effects (slope difference below $-20 \%$ ).

Omethoate and dimethoate were not much affected by matrix effects, together with diuron that only showed matrix suppression for mangostan. For several compounds, no signal suppression was observed for any of the matrices, and thus matrix effects, when present, led to only signal enhancement (methomyl, thiametoxam, 3-OH carbofuran, thiabendazol, imazalil, 3,4-dichloroaniline). It is worth to notice that imazalil and thiabendazol were affected by ionization enhancement in all matrices tested. The occurrence of important matrix effects for imazalil is in agreement with data reported by other authors [32].

On the contrary, for a few compounds only signal suppression was observed, although not in all matrices (paraoxon methyl, carbofuran, clomazone, parathion methyl, chlorpyrifos). Among these, parathion methyl and chlorpyrifos were affected by ionization suppression in nearly all matrices tested (11 out of 12, and 10 out of 12, respectively). Some other compounds were affected ion both ways, showing matrix suppression for some matrices and matrix enhancement for others (carbendazim, clothianidin, thiacloprid, malathion).

In relation to the matrix sample analyzed, some trends were observed. For example, guayaba, carambolo and tamarillo showed signal enhancement for almost all pesticides, while mangostan and feijoa led predominantly to signal suppression. The most difficult sample in terms of matrix effects was mangostan, where strong matrix effects were mostly observed.

The different behavior observed for compounds and food matrices under study showed that matrix effects were not homogeneous as a function of the chromatographic retention or the matrix analyzed, despite that some trends, as previously commented, were observed. Therefore, it seems not easy to predict the signal and extension of the matrix effects for each analyte/matrix combination.

\subsection{Method validation}

The usefulness of the approach used in this work was evaluated by calculating recoveries in fortified samples after applying the overall analytical procedure (i.e 
466 process efficiency, which includes the extraction process recovery and the influence of 467 matrix effects). Six matrices were selected and fortified (before extraction) at two 468 concentrations, 0.01 and $0.1 \mathrm{mg} / \mathrm{kg}$. Analyses were performed in quintuplicate, using 469 calibration curves with standards in solvent that were introduced at the beginning and 470 the end of each sequence of analysis of every matrix sample.

471 The process efficiency was calculated using the concentrations obtained by direct 472 calibration (standards in solvent) and also after application of the corrections factors 473 corresponding to the analyte/matrix combination under study (see Figure 3). Correction 474 factors were only applied when matrix effects were significant (i.e. differences in slopes 475 above $\pm 20 \%$; green and yellow color boxes in Figure 3 ), simulating the procedure that 476 would be applied in routine analysis.

477 Figure 4 shows the results obtained in this experiment for the six matrices 478 evaluated at the low concentration level tested $(0.01 \mathrm{mg} / \mathrm{kg})$. In general, the correction 479 was satisfactory for all matrices, leading to recoveries in the range $70-120 \%$, and data 480 were consistent at the two concentrations tested. It can be easily visualized the 481 satisfactory correction for granadilla for several pesticides whose recoveries were out 482 of the $70-120 \%$ range. However, after correction, recoveries reached the desired 483 values for up to 6 pesticides that were out of tolerance before correction (thiamethoxan, 484 clothianidin, thiabendazole, thiacloprid, paraoxon methyl, parathion methyl) (Figure 4a). 485 In tamarillo, 4 pesticides that were out of tolerances were satisfactorily corrected by 486 applying the correction factors (Figure $4 \mathrm{~b}$ ). The same occurred for 8 pesticides in 487 uchuva, the matrix for which correction was more significant (Figure 4c); 5 pesticides in 488 pithaya (Figure 4d); 5 in maracuya (Figure 4e) and 6 in gulupa (Figure 4f). Apart from a 489 few cases where the correction did not seem sufficient, the general trend was 490 satisfactory.. 3,4-dichloroaniline (metabolite of diuron) did consistently show recovery 491 values below $70 \%$ (mostly between 40 and $60 \%$ ) in four of the matrices tested 492 (granadilla, tamarillo, uchuva, pithaya). This might be explained because this analyte 493 may form strong bonds with common substances present in vegetable matrices and/or 494 due to partial degradation during the sample treatment, making its recovery poor with 495 common extraction methods. Other authors also reported recoveries around $60-70 \%$ 496 for this compound in the LC-UV determination of linuron and three metabolites $(3,4-$ 497 dichloroaniline included) in potatoes [53]. A few compounds (carbendazim in three 498 samples, and omethoate/ dimethoate in maracuya) could not be validated due to the 499 presence of the analyte in the "blank" sample used in method validation. Recovery data 500 obtained are shown in Table 2, S I. 
Other parameters included in method validation were linearity of the calibration curve for standards in solvent, precision (expressed as repeatability, from recovery experiments) and limits of detection. The linearity was tested between $2.5-500 \mathrm{ng} / \mathrm{mL}$ (equivalent to $0.0025-0.5 \mathrm{mg} / \mathrm{kg}$ in sample). It was satisfactory in the majority of cases (commonly up to $250 \mathrm{ng} / \mathrm{mL}$ ), with correlation coefficients above 0.99 and residuals lower than $\pm 20 \%$. The LOQ objective was established as the lowest concentration that was validated in a fortified sample after application of the overall analytical procedure. According to our data, the LOQ objective was $0.01 \mathrm{mg} / \mathrm{kg}$ for the wide majority of compounds (see Figure 4, and Table $2 \mathrm{SI}$ ), as satisfactory recoveries (70-120\%) and precision (RSD < 20\%) were obtained at this level. No chromatographic peaks were observed in the processed blank samples; therefore, LODs as low as $0.5-3.0 \mu \mathrm{g} / \mathrm{kg}$ $(0.0005$ and $0.003 \mathrm{mg} / \mathrm{kg}$ ) were estimated for a $\mathrm{S} / \mathrm{N}=3$ depending on the analyte/matrix combination.

In addition to the above indicated validation made for six selected matrices, the approach suggested in this work for matrix effects correction was supported by analysis of Quality Control (QC) samples that were included in the analysis sequence. QCs consisted on the same samples analyzed (12 samples from Spanish market and 12 samples collected directly from domestic Colombian markets) but previously fortified at 0.01 and $0.1 \mathrm{mg} / \mathrm{kg}$. Thus, QC recoveries were obtained for all sample matrices, included those that were not subjected to validation (i.e. papaya, guayaba, feijoa, mangostan, lulo and carambolo).In this way every sample was analyzed as a "blank" (without fortification) and after fortification at two concentration levels as QCs (see next section), as explained in the next section.

\subsection{Analysis of samples from the Spanish and Colombian markets}

24 samples were analyzed following the procedure developed in this work. Two samples were analyzed for each type of matrix: one collected in Spain (although imported from Colombia) and the other collected directly in Bogotá domestic markets. QCs recovery data at 0.01 and $0.1 \mathrm{mg} / \mathrm{kg}$ allowed us to know whether the analytical methodology applied was adequate and whether matrix effects correction, using the correction factors previously calculated, was satisfactory. This was especially important for the six tropical matrices that had not been previously validated, and whose overall recoveries had not been calculated.

Data for QCs are shown in Table 2. As being an individual value, the acceptance criterion was $60-140 \%$, in the line of the SANCO guideline for routine multi-residue analysis [39]. Among all QCs analysis, three individual recovery data were not 
537 available due to the presence of the analyte in the sample at relatively high 538 concentrations (these cases corresponded to carbendazim in papaya and lulo). So, 539 from 228 possible recovery data (corresponding to 19 compounds $\times 12$ matrices $\times 2$ 540 levels), up to 225 QCs recoveries were available. As it can be seen in Table 2, 202 out 541 of 225 data were within the acceptable range. This corresponded to $90 \%$ of QC 542 recoveries obtained. As expected from method validation for 3,4-dichloroaniline, 543 several QCs recoveries for this compound were out of tolerance (mangostan, lulo and 544 carambolo were around 40\%). Apart from this analyte, the exceptions were mostly 545 observed for methomyl and chlorpyrifos (5 data out of range), paraoxon methyl (4 data) 546 and thiabendazole (2 data). Data for QCs in analyses of real-world samples, together 547 with those obtained in method validation, support the applicability of the approach 548 proposed in this work for matrix effects correction.

549 In relation to the positives found in samples, Table 3 shows a summary of data 550 obtained. 18 detections were found in the 12 samples from Spanish markets 551 (emphasizing lulo sample with 5 positives and maracuyá with 3), while 16 detections 552 were observed in the 12 samples from Colombian domestic market (emphasizing 553 gulupa and carambolo with 3 positives each). In total, 9 different compounds were 554 detected, and corresponded to 4 insecticides (methomyl, dimethoate, thiacloprid, 555 carbofuran), 1 fungicide (carbendazim), 1 herbicide (diuron) and 3 metabolites 556 (omethoate, clothianidin, paraoxon methyl). As stated before, the LOQ objective was $5570.01 \mathrm{mg} / \mathrm{kg}$ as the method was not validated at concentrations below this value. 558 However, the sensitivity was sufficient to allow estimating concentrations in positive 559 samples far below the LOQ objective. In those cases, we could estimate the 560 concentration in sample as the signal obtained was above $\mathrm{S} / \mathrm{N}$ ratio of 10 , commonly 561 used as statistical LOQ of analytical methods. These values are marked by an asterisk 562 in Table 3.

563 The compound most frequently detected, and at higher concentrations, was 564 carbendazim that reached levels up to $3.4 \mathrm{mg} / \mathrm{kg}$ in papaya and was above $0.5 \mathrm{mg} / \mathrm{kg}$ 565 in lulo and granadilla. This fungicide was mostly present in samples collected in Spain, 566 which might imply that this compound was used as post-harvest fungicide during 567 storage and transport. Apart from carbendazim, the rest of compounds did not exceed $5680.1 \mathrm{mg} / \mathrm{kg}$ in samples, with the only exception of dimethoate in a maracuyá sample.

569 In Figure 5, several chromatograms for positive samples are shown as illustrative 570 examples. In all cases, two transitions were acquired and the $q / Q$ ion ratio was within 571 the tolerances admitted $( \pm 30 \%)$ supporting the reliable identification of the compound 572 detected in the sample. 
The strategy proposed in this work is of easy application to other laboratories, which should estimate their own correction factors after performing an evaluation of matrix effects under their experimental conditions (as shown in sections 2.5 and discussed in section 3.3). After around 5 months that passed from matrix effects evaluation (estimation of correction factors) and analysis of the samples collected at Colombia, the correction factors were successfully applied to the QCs analyzed, showing the robustness of this approach in our laboratory. Correction factors would need to periodically be checked for possible changes in the MS and chromatographic conditions, and as also for different varieties of each food product to ensure an appropriate correction.

\section{CONCLUSIONS}

Many multiresidue pesticide methods have been reported in the scientific literature for fruits and vegetables. However, few methods have been specifically addressed to tropical food, which may become a problem when assessing the compliance of Maximum Residue Levels in these products. In this work, twelve tropical fruits highly popular in Colombia, with increasing relevance in international trade markets (carambolo, feijoa, granadilla, guayaba, gulupa, lulo, mangostan, maracuya, papaya, pithaya, tamarillo, uchuva), have been selected for the LC-MS/MS determination of 20 pesticides and metabolites. After using a QuEChERS-based sample treatment with acetonitrile as extracting solvent, a detailed study was made on matrix effects associated to the LC-MS/MS analysis. A series of correction factors have been proposed for each analyte/matrix combination in order to facilitate the accurate quantification of the compounds using calibration standards in solvent. By application of appropriate correction factors there was no need for using either isotope-labeled internal standards or matrix-matched calibration in every sequence of sample analysis for matrix effects correction. The methodology developed has been validated at 0.01 and $0.1 \mathrm{mg} / \mathrm{kg}$ levels in six sample matrices, and the usefulness of correction factors was tested in the rest of matrices by evaluating recoveries of quality control samples included in every sequence of sample analysis. Analysis of samples collected in Spain (exported from Colombia) and directly in Bogota domestic markets revealed the presence of some of the compounds under study (mainly the fungicide carbendazim, the insecticide dimethoate and its metabolite omethoate, and the insecticide thiacloprid). With the exception of carbendazim (the maximum level found was 3.4 $\mathrm{mg} / \mathrm{kg}$ in a papaya sample), the rest of positives were below $0.2 \mathrm{mg} / \mathrm{kg}$, the majority of 
609 them being far below this value. MRLs set-up by the EU for these compounds [2] were

610 exceeded for carbendazim in four samples (papaya, lulo, granadilla and maracuya,

611 whose MRLs are between $0.1 \mathrm{mg} / \mathrm{kg}$ and $0.3 \mathrm{mg} / \mathrm{kg}$ ), for dimetoathe in one maracuya 612 sample (MRL $0.02 \mathrm{mg} / \mathrm{kg}$ ), and diuron in one uchuva sample (MRL $0.01 \mathrm{mg} / \mathrm{kg}$ ). It is 613 worth noting that MRLs for these tropical fruits are commonly set at the default value 614 corresponding to the limit of determination due to the lack of GLP studies on residue 615 trials for these matrices. This fact makes that even small concentrations of pesticides in 616 the samples may easily exceed the MRLs.

617

618

619

620 ACKNOWLEDGEMENTS

621 (Research Group of Excellence Prometeo 2009/054 and Prometeo II 2014/023;

622 Collaborative Research on Environment and Food-Safety, ISIC/2012/016).

623 


\section{References}

[1] FAO, Document BA/TF03/15. Committee on Commodity Problems, Intergovermental group of bananas and tropical fruits., (2003). http://www.fao.org/corp. Accesed Feb 2014.

[2] European Union Decision, Regulation (EC) N $N^{\circ} 396 / 2005$ of The European Parliament and of the council of 23 February 2005 on maximum residue levels of pesticides in or on food and feed of plant and animal origin and amending Council Directive 91/414/EEC.

[3] Exportación de frutas exóticas colombianas. Legiscomex, (2013). www.legiscomex.com/bancomedios/documentos/estudio_frutas_tropicale s_Colombia. Accesed Jun 2014.

[4] Botitsi H V, Garbis SD, Economou A, Tsipi DF (2011) Mass Spectrom Rev 30: 907-939.

[5] Alder L, Greulich K, Kempe G, Vieth B, (2006) Mass Spectrom. Rev. 25: 838-865.

[6] Hernández F, Sancho JV, Pozo OJ (2005) Anal. Bioanal. Chem. 382: 934-946.

[7] Hernández F, Pozo OJ, Sancho JV, Bijlsma L, Barreda M, Pitarch E, (2006) J. Chromatogr. A. 1109: 242-252.

[8] Pico Y, Vale D (2006) Mass Spectrom. Rev. 25:917-960.

[9] Kmellár B, Fodor P, Pareja L, Ferrer C, Martínez-Uroz M, Valverde A, Fernandez-Alba AR (2008) J. Chromatogr. A. 1215: 37-50.

[10] Camino-Sánchez FJ., Zafra-Gómez A, Oliver-Rodríguez B, Ballesteros O, Navalón A, Crovetto G, Vilchez JL (2010) Food Addit. Contam. Part A. 27: 1532-1544.

[11] Grimalt S., Sancho JV, Pozo OJ., Hernández F (2010) J. Mass Spectrom. 45:421-436.

[12] Kmellár B, Abrankó L, Fodor P, Lehotay SJ (2010) Food Addit. Contam. Part A. 27:1415-1430.

[13] Marín JM, Gracia-Lor E, Sancho JV, López FJ, Hernández F (2009) J. Chromatogr. A. 1216: 1410-1420.

[14] Soler C, Mañes J, Picó Y (2008) Crit. Rev. Anal. Chem. 38: 93-117.

[15] Gebara AB., Ciscato C, Ferreira M, Monteiro S (2005) Bull. Environ. Contam. Toxicol. 75: 163-169. 
[16] Ciscato CHP, Gebara AB, Monteiro SH (2009) Food Addit. Contam. Part B. 2: $140-145$.

[17] Specht W (1992) Manual of pesticide residue analysis, DFG Deutsche Forschungsgemeinschaft, Pesticides Commission. VCH, Weinheim, pp 317-322.

[18] Banerjee K, Oulkar DP, Patil SB, Jadhav MR, Dasgupta S, Patil SH, Bal S, Adsule PG (2009) J. Agric. Food Chem. 57: 4068-4078.

[19] Savant RH, Banerjee K, Utture SC, Patil SH, Dasgupta S, Ghaste MS, Adsule PG (2010) J. Agric. Food Chem. 58: 1447-1454.

[20] Navickiene S, Aquino A, Bezerra DSS (2010) J. Chromatogr. Sci. 48:750754.

[21] Sundravadana S, Alice D, Samiyappan R, Kuttalam S (2008) J. Braz. Chem. 19: 60-63.

[22] Hjorth K, Johansen K, Holen B, Andersson A, Christensen HB, Siivinen K, Toome M. (2011) Food Control. 22: 1701-1706.

[23] Ramírez A, Gallo AF, Hoyos DE, Peñuela GA (2014) Food Chem. 158:153-161.

[24] Taylor PJ (2005) Clin. Biochem. 38:328-34.

[25] Niessen WMA, Manini P, Andreoli R (2006) Mass Spectrom. Rev. 25:881-899.

[26] Gosetti F, Mazzucco E, Zampieri D, Gennaro MC (2010) J. Chromatogr. A. 1217:3929-3937.

[27] Trufelli H, Palma P, Famiglini G, Cappiello A (2011), Mass Spectrom. Rev. 30:491-509.

[28] Cappiello A, Famiglini G, Palma P, Trufelli H (2010) J. Liq. Chromatogr. Relat. Technol. 33:1067-1081.

[29] González-Antuña A, Domínguez-Romero JC, García-Reyes JF, Rodríguez-González P, Centineo G, García Alonso JI, Molina-Diaz A (2013) J. Chromatogr. A. 1288:40-47.

[30] Hewavitharana AK (2011) J. Chromatogr. A. 1218:359-61.

[31] Lucini L, Pietro Molinari G (2011) J. Chromatogr. Sci. 49:709-714.

[32] Ferrer C, Lozano A, Agüera A, Girón AJ, Fernández-Alba AR (2011) J. Chromatogr. A. 1218:7634-7639. 
691 [33] Stachniuk A, Fornal E (2013) Cent. Eur. J. Chem. 11:1112-1131.

692 [34] Kwon H, Lehotay SJ, Geis-Asteggiante L (2012) J. Chromatogr. A. 693

694

695

696

697

698

699

700

701

702

703

704

705

706

707

708

709

710

711

712

713

714

715

716

717

718

719

720

721

722

723

[35] Kruve A, Künnapas A, Herodes K, Leito I (2008) J. Chromatogr. A. 1187:58-66.

[36] Chung SWC, Chan BTP (2010) J. Chromatogr. A. 1217:4815-4824.

[37] Kittlaus S, Kempe G, Speer K (2013) J. Sep. Sci. 36:2185-2195.

[38] Botero-Coy AM, Marín JM, Ibáñez M, Sancho JV, Hernández F (2012) Anal. Bioanal. Chem. 402:2287-2300.

[39] Method validation and quality control procedures for pesticide residues analysis in food and feed. Document SANCO/12571/2013 (2014).

[40] Pozo ÓJ, Sancho JV, Ibáñez M, Hernández F, Niessen WMA (2006) Trends Anal. Chem. 25:1030-1042.

[41] Anastassiades M, Lehotay SJ, Stajnbaher D, Schenck FJ (2003) J. AOAC Int. 86:412-431.

[42] Payá P, Anastassiades M, Mack D, Sigalova I, Tasdelen B, Oliva J, Barba A (2007) Anal. Bioanal. Chem. 389:1697-1714.

[43] Wilkowska A, Biziuk M (2011) Food Chem. 125:803-812.

[44] Lehotay SJ, Son KA, Kwon H, Koesukwiwat U, Wusheng Fu, Mastovska K, Hoh E, Leepipatpiboon N (2010) J. Chromatogr. A. 1217:2548-2560.

[45] Lehotay SJ (2007) J. AOAC Int. 90:485-520.

[46] CEN, UNE-EN 15662, July 2008. Foods of plant origin. Determination of pesticide residues using GC-MS and/or LC-MS/MS following acetonitrile extraction/partitioning and clean-up by dispersive SPE-QUECHERSmethod.

[47] España Amórtegui JC, Guerrero Dallos JA (2014) Food Addit. Contam. Part A. 31:676-687.

[48] Lehotay SJ, Anastassiades M, Majors RE (2010) Chromatography Online http://www.chromatographyonline.com/ (accesed March 2014).

[49] Romero-González R, Aguilera-Luiz MM, Plaza-Bolaños P, Frenich AG, Vidal JLM (2011) J. Chromatogr. A. 1218:9353-9365.

[50] Schenck FJ, Brown AN, Podhorniak LV, Parker A, Reliford M, Wong JW (2008) J. AOAC Int. 91:422-438. 
724 [51] Acidic pesticides using QuEChERS method; http://quechers.cvuastuttgart.de/pdf/acidicpesticides.pdf (accessed January 2015)

726 [52] Romero-González R, Garrido A, Martínez Vidal JL, Prestes OD, Grio SL 727 (2011) J. Chromatogr. A. 1218:1477-1485.

728

729

[53] Miliadis GE, Siskos PA, Vasilikiotis GS (1990) J. Assoc. Off. Anal. Chem. 73:435-437.

730

731 


\section{Figure captions}

Figure 1. Tropical Fruits studied in this work and MRLs groups as classified in Annex I "Products of plant and animal origin" [2]

Figure 2. Calibration graphs obtained for selected pesticides in different sample matrices. Calibration in solvent and $\pm 20 \%$ tolerance in the slope is highlighted in yellow

Figure 3. Difference (\%) between matrix calibration and direct calibration slopes. Correction factors (in brackets) were applied only when difference was higher than $\pm 20 \%$ (green/dark boxes and yellow/light boxes).

Figure 4. Average recoveries for selected pesticides in different sample matrices after application of the overall analytical procedure. Recovery data correspond to samples spiked at 0.01 and $0.1 \mathrm{mg} / \mathrm{kg}$, and were calculated using calibration with standards in solvent with and without application of correction factors (see Figure 3 for correction factors)

Figure 5. Illustrative chromatograms for pesticides detected in tropical fruit samples. $Q$ quantification transition. q confirmation transition. $q / Q$ ratios in samples were within the maximum tolerances admitted in relation with those of reference standards 
Table 1. MS/MS optimized conditions (ESI+) for the compounds and metabolites studied in this work.

\begin{tabular}{|c|c|c|c|c|c|c|}
\hline Compound & $\begin{array}{c}t R \\
(\min )\end{array}$ & $\begin{array}{l}\text { Precursor } \\
\text { ion }(\mathrm{m} / \mathrm{z})\end{array}$ & $\begin{array}{c}\text { Cone } \\
(\mathrm{V})\end{array}$ & $\begin{array}{c}\text { Collision } \\
\text { Energy } \\
(\mathrm{eV})\end{array}$ & $\begin{array}{l}\text { Product } \\
\text { ion }(\mathrm{m} / \mathrm{z})\end{array}$ & $\begin{array}{l}\mathrm{q} / \mathbf{Q} \\
\text { Ratio }\end{array}$ \\
\hline $\begin{array}{l}\text { Omethoate } \\
\text { (OME) }\end{array}$ & 5.70 & 214.3 & 25 & $\begin{array}{l}10 \\
15\end{array}$ & $\begin{array}{c}183.1 \\
155\end{array}$ & 0.92 \\
\hline $\begin{array}{l}\text { Carbendazim } \\
\text { (CAR) }\end{array}$ & 6.75 & 192.1 & 30 & $\begin{array}{l}15 \\
30\end{array}$ & $\begin{array}{l}160.1 \\
132.0\end{array}$ & 0.17 \\
\hline $\begin{array}{l}\text { Methomyl } \\
\text { (MTL) }\end{array}$ & 6.98 & 163.1 & 20 & $\begin{array}{l}10 \\
10\end{array}$ & $\begin{array}{c}88.0 \\
106.0\end{array}$ & 0.77 \\
\hline $\begin{array}{l}\text { Thiametoxam } \\
\text { (THI) }\end{array}$ & 7.26 & 292.0 & 25 & $\begin{array}{l}15 \\
25\end{array}$ & $\begin{array}{l}211.2 \\
181.2\end{array}$ & 0.66 \\
\hline $\begin{array}{l}\text { Thiabendazole } \\
\text { (THB) }\end{array}$ & 7.56 & 202.3 & 35 & $\begin{array}{l}25 \\
30\end{array}$ & $\begin{array}{l}175.1 \\
131.2\end{array}$ & 0.63 \\
\hline $\begin{array}{l}\text { Picloram } \\
\text { (PIC) }\end{array}$ & 7.87 & 241.1 & 25 & $\begin{array}{l}20 \\
30\end{array}$ & $\begin{array}{l}195.2 \\
168.0\end{array}$ & 0.58 \\
\hline $\begin{array}{l}\text { Clothianidin } \\
\text { (CLOT) }\end{array}$ & 8.21 & 250.2 & 30 & $\begin{array}{l}15 \\
15\end{array}$ & $\begin{array}{l}169.2 \\
132.0\end{array}$ & 0.65 \\
\hline $\begin{array}{l}\text { 3-Hydroxycarbofuran } \\
\qquad(3-\mathrm{OH})\end{array}$ & 8.38 & 238.3 & 30 & $\begin{array}{l}10 \\
15\end{array}$ & $\begin{array}{l}181.2 \\
163.1\end{array}$ & 0.80 \\
\hline $\begin{array}{l}\text { Dimethoate } \\
\quad \text { (DIM) }\end{array}$ & 8.48 & 230.1 & 25 & $\begin{array}{l}10 \\
20\end{array}$ & $\begin{array}{l}199.1 \\
125.0\end{array}$ & 0.77 \\
\hline $\begin{array}{l}\text { Thiacloprid } \\
\text { (THC) }\end{array}$ & 9.07 & 253.2 & 35 & $\begin{array}{l}20 \\
40\end{array}$ & $\begin{array}{l}126.0 \\
90.0\end{array}$ & 0.21 \\
\hline $\begin{array}{l}\text { Paraoxon Methyl } \\
\text { (PXON) }\end{array}$ & 9.59 & 248.2 & 40 & $\begin{array}{l}20 \\
35\end{array}$ & $\begin{array}{l}202.2 \\
127.0\end{array}$ & 0.10 \\
\hline $\begin{array}{l}\text { Imazalil } \\
\text { (IMA) }\end{array}$ & 9.63 & $\begin{array}{l}297.2 \\
299.2\end{array}$ & 35 & $\begin{array}{l}25 \\
20\end{array}$ & $\begin{array}{l}159.1 \\
161.1\end{array}$ & 0.73 \\
\hline $\begin{array}{l}\text { Carbofuran } \\
\text { (CRB) }\end{array}$ & 9.98 & 222.2 & 30 & $\begin{array}{l}10 \\
20\end{array}$ & $\begin{array}{l}165.1 \\
123.1\end{array}$ & 0.79 \\
\hline
\end{tabular}




\begin{tabular}{|c|c|c|c|c|c|c|}
\hline $\begin{array}{l}\text { Malaoxon } \\
(\text { MLX) }\end{array}$ & 10.08 & 315.2 & 30 & $\begin{array}{l}15 \\
10\end{array}$ & $\begin{array}{l}127.1 \\
143.0\end{array}$ & 0.15 \\
\hline $\begin{array}{c}\text { 3,4-dichlooraniline } \\
\text { (3,4 DCA) }\end{array}$ & 10.56 & $\begin{array}{l}162.1 \\
164.1\end{array}$ & 30 & $\begin{array}{l}20 \\
20\end{array}$ & $\begin{array}{l}127.0 \\
129.0\end{array}$ & 0.33 \\
\hline $\begin{array}{c}\text { Diuron } \\
\text { (DIU) }\end{array}$ & 11.06 & $\begin{array}{l}233.1 \\
235.2\end{array}$ & 35 & $\begin{array}{l}15 \\
10\end{array}$ & $\begin{array}{l}71.9 \\
71.9\end{array}$ & 0.31 \\
\hline $\begin{array}{l}\text { Clomazone } \\
\text { (CLO) }\end{array}$ & 11.19 & $\begin{array}{l}240.2 \\
242.2\end{array}$ & 30 & $\begin{array}{l}20 \\
20\end{array}$ & $\begin{array}{l}125.1 \\
127.1\end{array}$ & 0.32 \\
\hline $\begin{array}{c}\text { Parathion- methyl } \\
\text { (PAR) }\end{array}$ & 11.44 & 264.2 & 40 & $\begin{array}{l}15 \\
20\end{array}$ & $\begin{array}{l}125.1 \\
143.2\end{array}$ & 0.10 \\
\hline $\begin{array}{l}\text { Malathion } \\
\text { (MAL) }\end{array}$ & 11.69 & 331.1 & 30 & $\begin{array}{l}15 \\
10\end{array}$ & $\begin{array}{l}127.0 \\
285.0\end{array}$ & 0.43 \\
\hline $\begin{array}{c}\text { Chlorpyrifos } \\
\text { (CHLOR) }\end{array}$ & 13.37 & $\begin{array}{l}350.0 \\
352.0\end{array}$ & 30 & $\begin{array}{l}20 \\
20\end{array}$ & $\begin{array}{l}198.1 \\
200.1\end{array}$ & 0.97 \\
\hline
\end{tabular}


Table 2. Recoveries (\%) obtained for quality controls (at 0.01 and $0.1 \mathrm{mg} / \mathrm{kg}$ level) that were analyzed in the sample sequence for six tropical fruit matrices. Concentrations calculated using calibration standards in solvent and applying the corresponding correction factors (see Figure 3 )

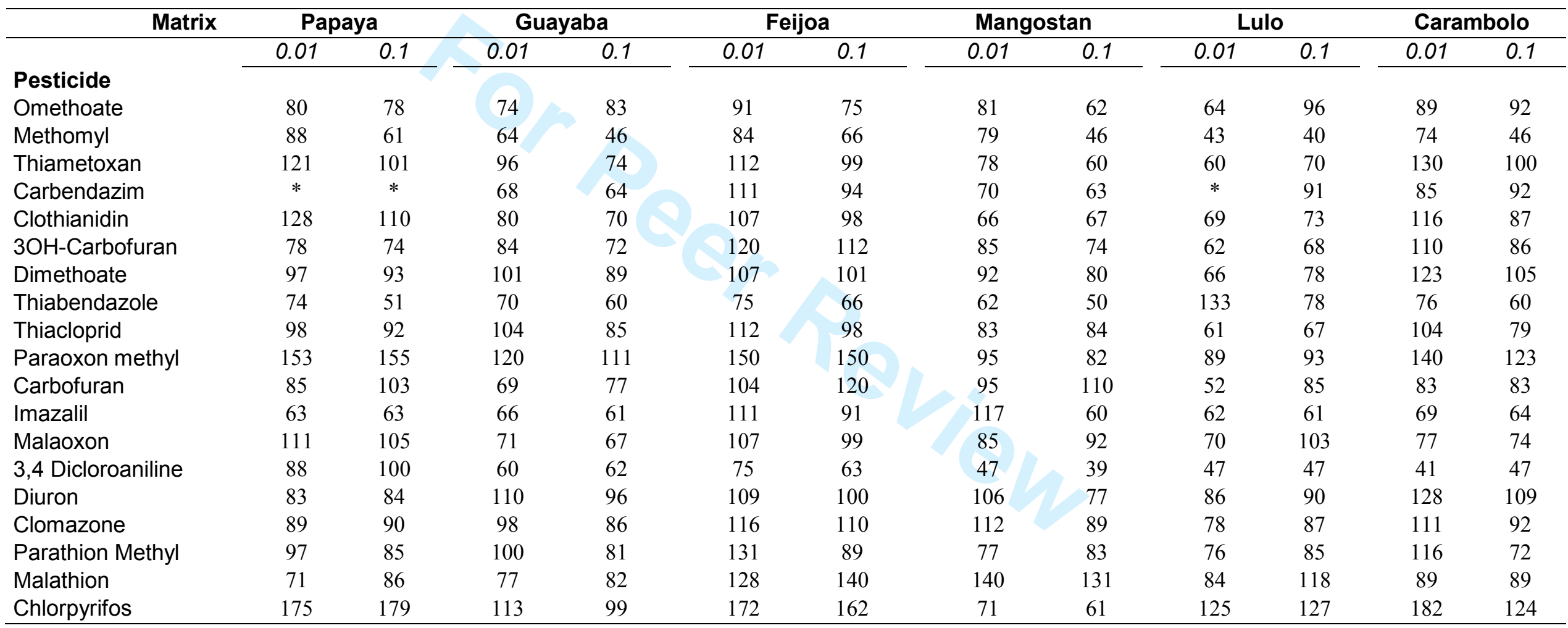

*Not determined because of the presence of analyte in unfortified sample 
Table 3. Pesticide concentrations $(\mu \mathrm{g} / \mathrm{kg})$ in the tropical fruits analyzed: (1) samples used for validation, collected from Spanish markets, (2) samples collected from Colombian domestic markets

\begin{tabular}{|c|c|c|c|c|c|c|c|c|c|c|c|c|}
\hline Pesticide & Lulo & Carambolo & Granadilla & Mangostan & Tamarillo & Gulupa & Maracuya & Uchuva & Guayaba & Pithaya & Papaya & Feijoa \\
\hline Omethoate & $2.9(1)^{\star}$ & - & - & - & $2.1(1)^{*}$ & $d(2)$ & $42(1)$ & - & - & - & - & - \\
\hline Methomyl & $1.5(1)^{*}$ & - & - & - & - & - & - & - & - & - & - & - \\
\hline Carbendazim & $\begin{array}{c}1340(1) \\
19(2) \\
\end{array}$ & $\begin{array}{c}2.1(1)^{*} \\
d(2)\end{array}$ & $\begin{array}{c}660(1) \\
- \\
\end{array}$ & $\begin{array}{c}3.5(1)^{*} \\
-\end{array}$ & $\begin{array}{c}290(1) \\
80(2) \\
\end{array}$ & $\begin{array}{l}7.2(1)^{*} \\
2.6(2)^{*}\end{array}$ & $\begin{array}{c}210(1) \\
30(2) \\
\end{array}$ & - & $\begin{array}{l}\mathrm{d}(1) \\
\mathrm{d}(2)\end{array}$ & $\begin{array}{c}- \\
d(2)\end{array}$ & $\begin{array}{c}3400(1) \\
- \\
\end{array}$ & $\begin{array}{c}\mathrm{d}(1) \\
3.2(2)^{*}\end{array}$ \\
\hline Clothianidin & - & - & - & - & $2.2(2)^{\star}$ & - & - & - & - & - & - & - \\
\hline Dimethoate & $1.6(1)^{*}$ & $10(2)$ & - & - & - & $2.0(2)^{*}$ & $160(1)$ & - & - & - & - & - \\
\hline Thiacloprid & $8.0(1)^{*}$ & $\mathrm{~d}(2)$ & - & - & - & - & - & - & - & - & - & - \\
\hline Carbofuran & $d(2)$ & - & - & - & - & - & - & - & - & - & - & $d(2)$ \\
\hline & & & & & & 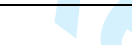 & & & & & & \\
\hline Diuron & - & - & - & - & - & - & - & $50(2)$ & - & - & - & - \\
\hline Paraoxon Methyl & - & - & - & - & - & - & - & - & - & - & - & $14(1)$ \\
\hline
\end{tabular}

$\mathrm{d}$ : detected

*: estimated concentration corresponding to a response above S/N ratio of 10 (below the LOQ objective of $0.01 \mathrm{mg} / \mathrm{kg}$ ). 


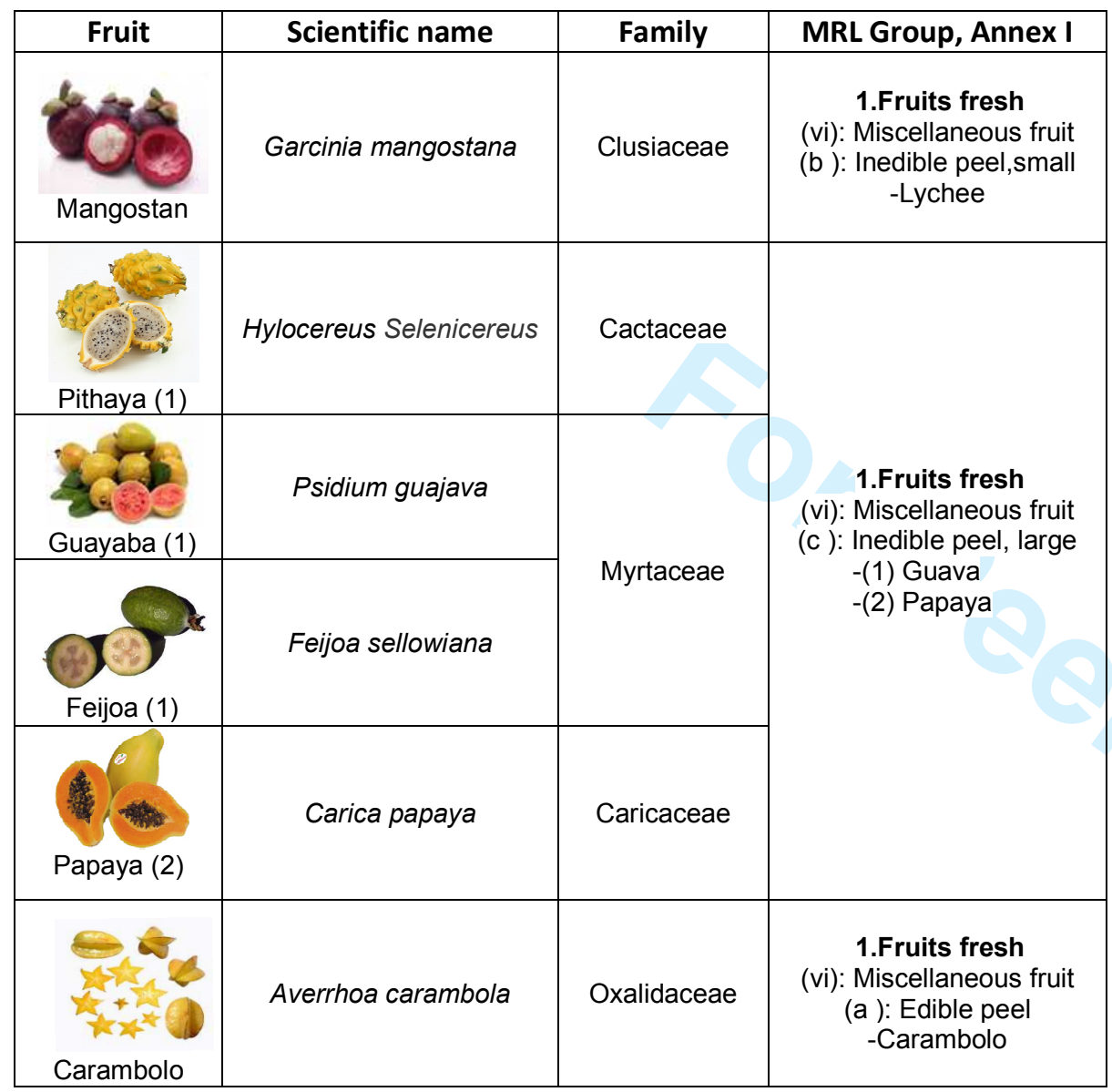

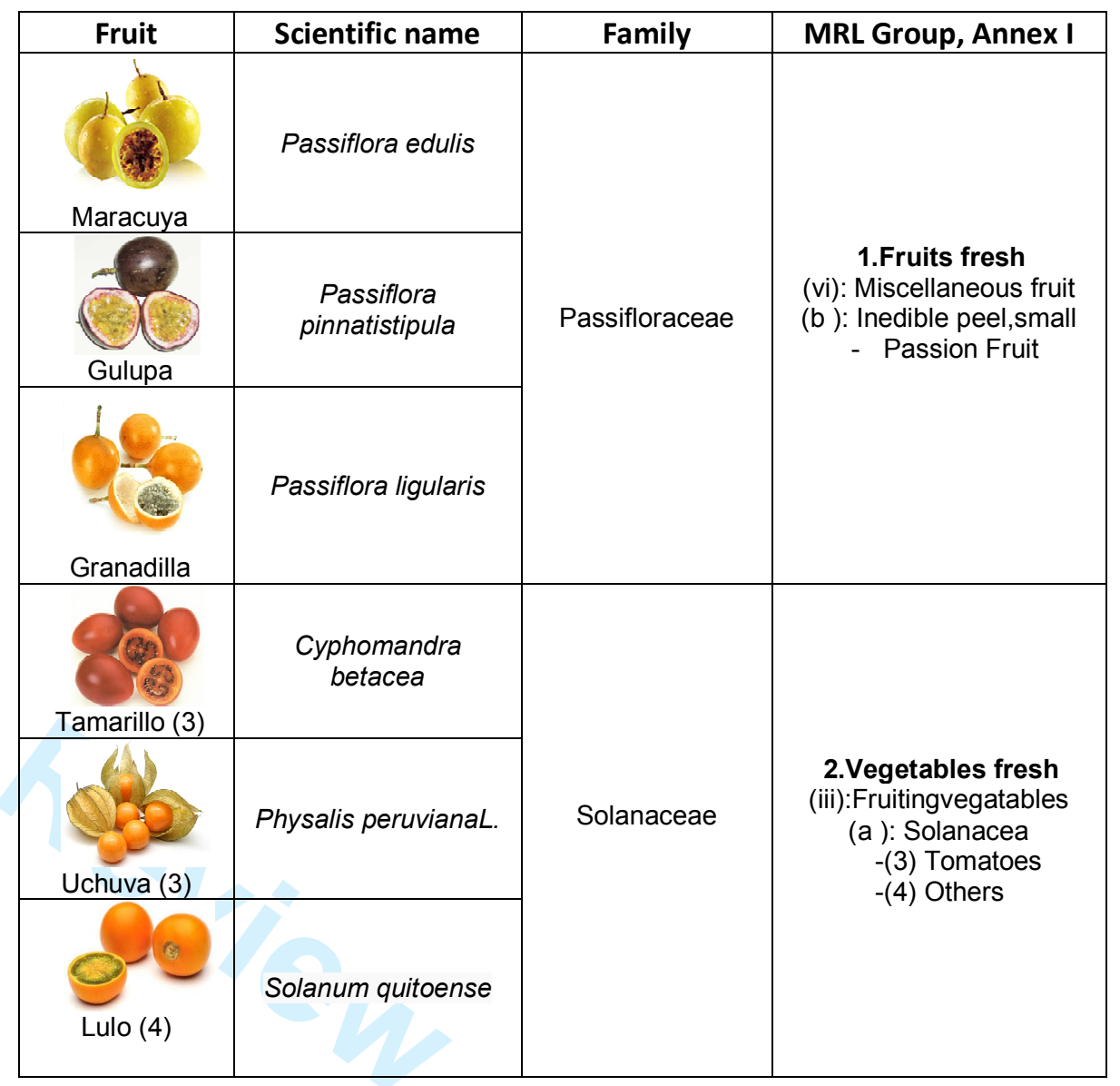

\section{Figure 1}


2

$\begin{array}{ll}3 & 5000 \\ 4 & 4500 \\ 5 & 4000\end{array}$

$\begin{array}{ll}4 & 4500 \\ 5 & 4000\end{array}$

63500

3000

2000

$10_{1500}$

$11_{1000}$

12500

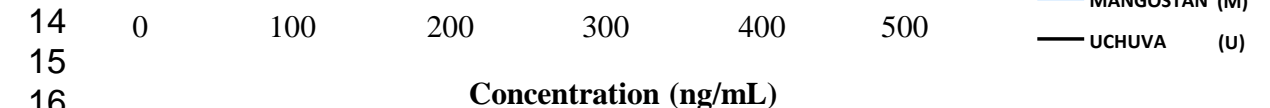

16

17

18

19
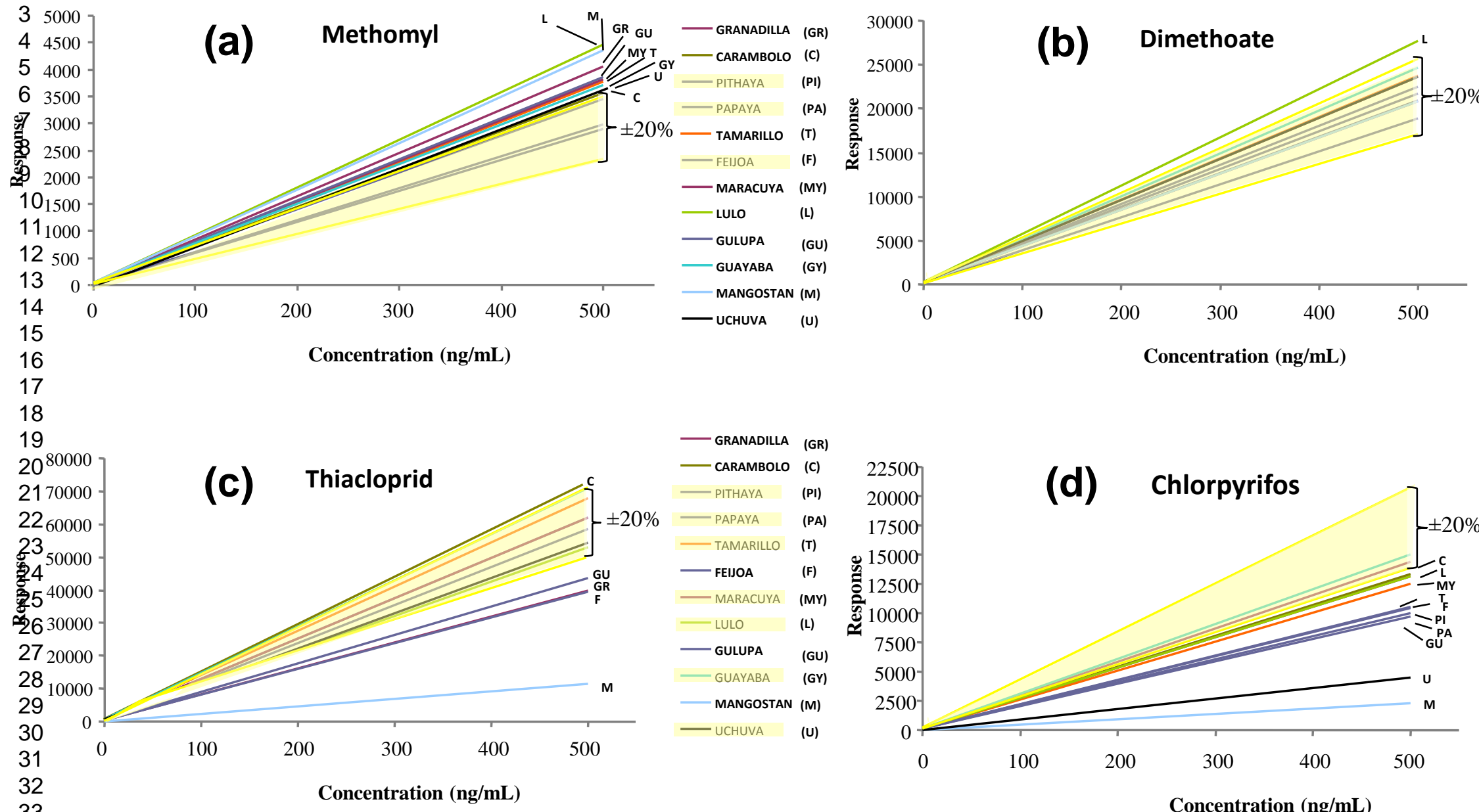

- GRANADILLA (GR) - CARAMBolo (C) CARAmbolo (C) $\begin{array}{ll}\text { - PAPAYA } & \text { (PA) } \\ \text { TAMARILLO } & \text { (T) } \\ \text { feIJOA } & \text { (F) }\end{array}$ - MARACUYA (MY) LULO (L) - GULUPA (GU) - GUAYABa (GY) - MANGOSTAN (M)

Concentration $(\mathbf{n g} / \mathrm{mL})$

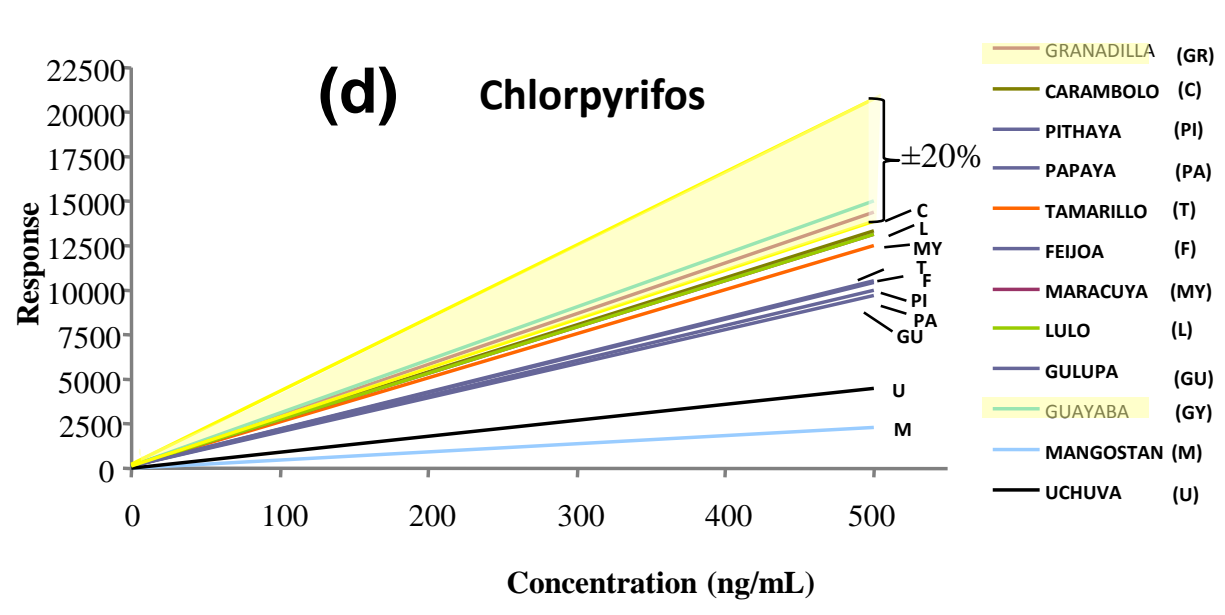

Figure 2 


\begin{tabular}{|c|c|c|c|c|c|c|c|c|c|c|c|c|}
\hline Compound & Granadilla & Lulo & Guayaba & Mangostan & Uchuva & Maracuya & Pithaya & Carambolo & Gulupa & Papaya & Tamarillo & Feijoa \\
\hline Omethoate & $11(0.90)$ & $-11(1.12)$ & $-10(1.11)$ & $-7(1.08)$ & $-2(1.02)$ & $20(0.83)$ & $-6(1.06)$ & $-3(1.03)$ & $13(0.88)$ & $-11(1.12)$ & $17(0.85)$ & $-8(1.09)$ \\
\hline \begin{tabular}{|l|} 
Methomyl \\
\end{tabular} & $39(0.72)$ & $53(0.65)$ & \begin{tabular}{|l|}
$27(0.79)$ \\
\end{tabular} & $49(0.67)$ & $26(0.79)$ & $31(1.45)$ & $18(0.85)$ & $22(0.82)$ & $32(0.76)$ & $2(0.98)$ & $29(0.78)$ & $-1(1.01)$ \\
\hline Thiametoxam & $35(0.74)$ & $30(0.77)$ & \begin{tabular}{|l|}
$32(0.76)$ \\
\end{tabular} & $22(0.82)$ & $54(0.65)$ & $38(0.72)$ & $42(0.70)$ & $22(0.82)$ & $-3(1.03)$ & $7(0.93)$ & $28(0.78)$ & $9(0.92)$ \\
\hline Carbendazim & $-18(1.22)$ & \begin{tabular}{|l}
$-55(2.22)$ \\
\end{tabular} & \begin{tabular}{|l|}
$26(0.79)$ \\
\end{tabular} & \begin{tabular}{|l|}
$24(0.81)$ \\
\end{tabular} & $-15(1.18)$ & $-22(1.28)$ & $18(1.22)$ & \begin{tabular}{|l}
$-8(1.09)$ \\
\end{tabular} & \begin{tabular}{|l}
$-16(1.19)$ \\
\end{tabular} & $-58(2.38)$ & $10(0.91)$ & $-12(1.14)$ \\
\hline Clothianidin & $-31(1.45)$ & $-2(0.79)$ & $23(0.81)$ & $-25(1.33)$ & $11(0.90)$ & $13(0.88)$ & $-15(1.18)$ & \begin{tabular}{|l|}
$36(0.74)$ \\
\end{tabular} & $-1(1.01)$ & $11(0.90)$ & $15(0.87)$ & $\begin{array}{l}-7(1.08) \\
\end{array}$ \\
\hline $3 \mathrm{OH}$ Carbofuran & $18(0.85)$ & $53(0.65)$ & \begin{tabular}{|l|}
$29(0.78)$ \\
\end{tabular} & \begin{tabular}{|l}
$25(0.80)$ \\
\end{tabular} & $32(0.76)$ & $21(0.83)$ & $18(0.85)$ & $24(0.81)$ & $14(0.88)$ & $22(0.82)$ & $26(0.79)$ & $4(0.96)$ \\
\hline Dimethoate & $-3(1.03)$ & $29(0.78)$ & $15(0.87)$ & $-3(1.03)$ & $10(0.91)$ & $10(0.91)$ & $5(0.95)$ & $10(0.91)$ & $-2(1.02)$ & $1(0.99)$ & $11(0.90)$ & $-12(1.14)$ \\
\hline Thiabendazol & $145(0.41)$ & $94(0.52)$ & $\begin{array}{l}153(0.40) \\
\end{array}$ & $\begin{array}{l}103(0.49) \\
\end{array}$ & $145(0.41)$ & $53(0.65)$ & $178(0.36)$ & $221(0.31)$ & $37(0.73)$ & $169(0.37)$ & $155(0.40)$ & $153(0.40)$ \\
\hline \begin{tabular}{|l|} 
Thiacloprid \\
\end{tabular} & $-33(1.49)$ & $-11(1.12)$ & $19(0.84)$ & $-81(5.26)$ & $-9(1.10)$ & $4(0.96)$ & $-2(1.02)$ & $21(0.83)$ & $-27(1.37)$ & $4(0.96)$ & $14(0.88)$ & $-34(1.52)$ \\
\hline Paraoxon methyl & $-37(1.59)$ & $-10(1.11)$ & $7(0.93)$ & \begin{tabular}{|l}
$-74(3.85)$ \\
\end{tabular} & $-20(1.25)$ & $-11(1.12)$ & $-25(1.33)$ & $0(1.0)$ & $\begin{array}{l}-53(2.13) \\
\end{array}$ & $-34(1.52)$ & $-8(1.09)$ & $-43(1.75)$ \\
\hline Carbofuran & $-17(1.20)$ & $-10(1.11)$ & $\begin{array}{l}-14(1.22) \\
\end{array}$ & \begin{tabular}{|l}
$-51(2.04)$ \\
\end{tabular} & $-30(1.43)$ & $-12(1.14)$ & $-11(1.12)$ & $0(1.0)$ & \begin{tabular}{|l}
$-17(1.20)$ \\
\end{tabular} & $-26(1.35)$ & $-18(1.22)$ & $\begin{array}{l}-26(0.85) \\
\end{array}$ \\
\hline Imazalil & $36(0.74)$ & $\begin{array}{l}119(0.46) \\
\end{array}$ & \begin{tabular}{|l}
$94(0.52)$ \\
\end{tabular} & \begin{tabular}{|l|}
$21(0.83)$ \\
\end{tabular} & $54(0.65)$ & $46(0.68)$ & $45(0.69)$ & \begin{tabular}{|l}
$103(0.49)$ \\
\end{tabular} & \begin{tabular}{|l|}
$23(0.82)$ \\
\end{tabular} & $89(0.53)$ & $53(0.65)$ & $\begin{array}{l}34(0.74) \\
\end{array}$ \\
\hline Malaoxo & $-2(1.02)$ & $-21(1.27)$ & $-11(1.12)$ & -33 & $-21(1.27)$ & -24 & 01) & -17 & -15 & $-39(1.64)$ & .25) & $-29(1.41)$ \\
\hline 3.4 Dichloroaniline* & $41(0.71)$ & $27(0.79)$ & $27(0.79)$ & $12(0.89)$ & $0(1.0)$ & $26(0.79)$ & $29(0.78)$ & $38(0.72)$ & $11(0.90)$ & $16(0.86)$ & $23(0.81)$ & $14(0.88)$ \\
\hline Diuron & $17(0.85)$ & $11(0.90)$ & $10(0.90)$ & \begin{tabular}{|l|}
$-50(2.0)$ \\
\end{tabular} & $-7(1.08)$ & $12(0.89)$ & $-12(1.14)$ & $16(0.86)$ & \begin{tabular}{|l|}
$-2(1.02)$ \\
\end{tabular} & $-16(1.19)$ & $9(0.92)$ & $-13(1.15)$ \\
\hline Clomazone & $6(0.94)$ & $9(0.92)$ & $10(0.91)$ & $-57(2.33)$ & $-50(2.0)$ & $4(0.96)$ & $-12(1.14)$ & $9(0.92)$ & $-7(1.08)$ & $3(0.97)$ & $5(0.95)$ & $-22(1.28)$ \\
\hline Parathion methyl & $-40(1.69)$ & $-23(1.30)$ & \begin{tabular}{|l}
$-30(1.43)$ \\
\end{tabular} & \begin{tabular}{|l}
$-66(2.94)$ \\
\end{tabular} & $-34(1.52)$ & $-21(1.27)$ & $-25(1.33)$ & \begin{tabular}{|l}
$-16(1.19)$ \\
\end{tabular} & \begin{tabular}{|l}
$-47(1.89)$ \\
\end{tabular} & $-38(1.61)$ & $-26(1.35)$ & $\begin{array}{l}-44(1.79) \\
\end{array}$ \\
\hline Malathion & $23(0.81)$ & $6(0.94)$ & $45(0.69)$ & \begin{tabular}{|l}
$-53(2.13)$ \\
\end{tabular} & $15(0.87)$ & $28(0.78)$ & 10 & \begin{tabular}{|l}
$28(0.78)$ \\
\end{tabular} & $22(0.82)$ & $23(0.81)$ & $.85)$ & $9(0.92)$ \\
\hline Chlorpyrifos & $-17(1.20)$ & $-24(1.16)$ & $-13(1.15)$ & \begin{tabular}{|l|}
$-87(7.69)$ \\
\end{tabular} & $-74(3.85)$ & $-24(1.32)$ & $-40(1.67)$ & $-23(1.30)$ & $-44(1.30)$ & $-42(1.72)$ & $-28(1.39)$ & $-39(1.64)$ \\
\hline
\end{tabular}

Figure 3 


\section{Page 33 of 52}

Analytical \& Bioanalytical Chemistry

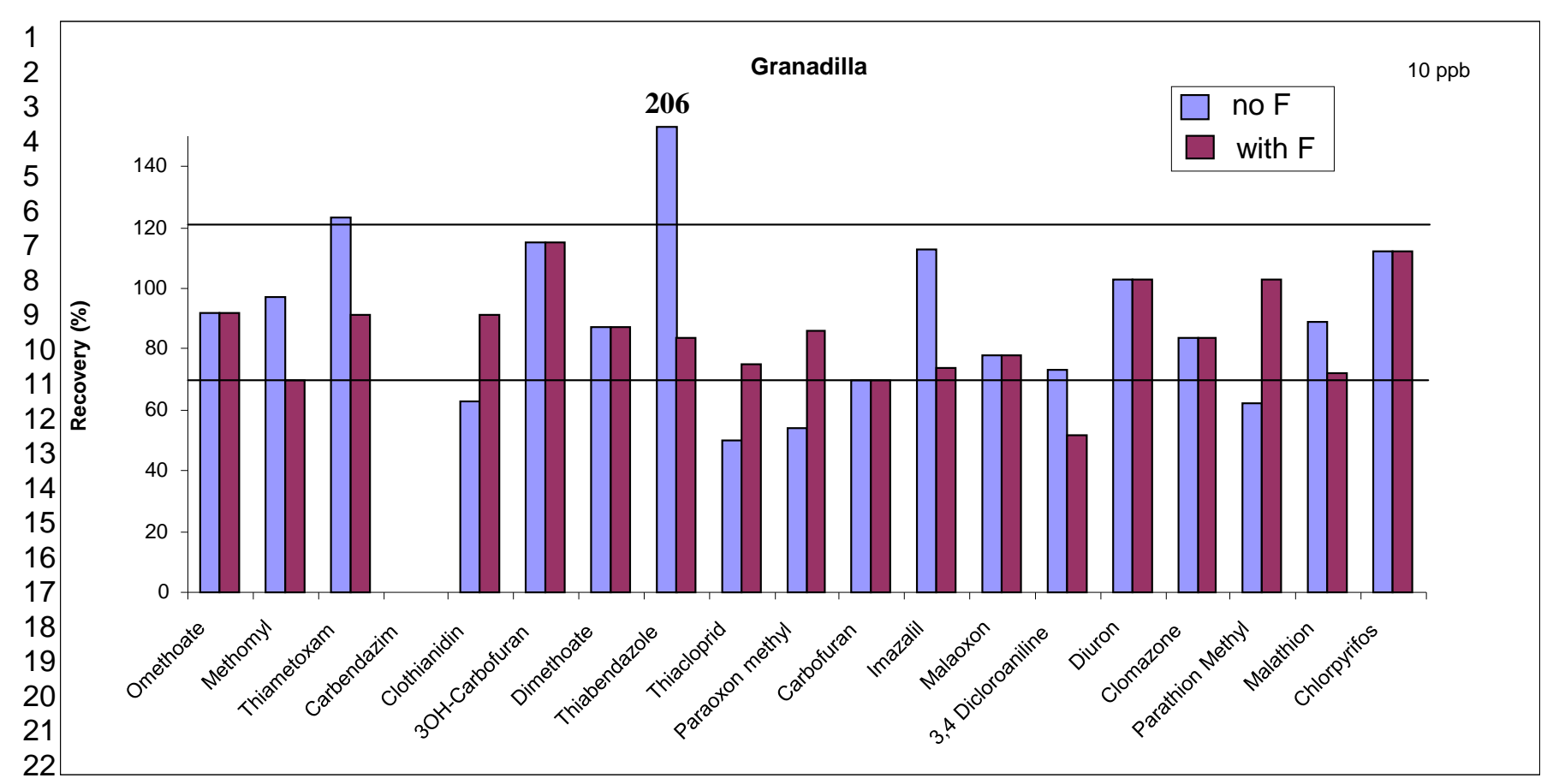



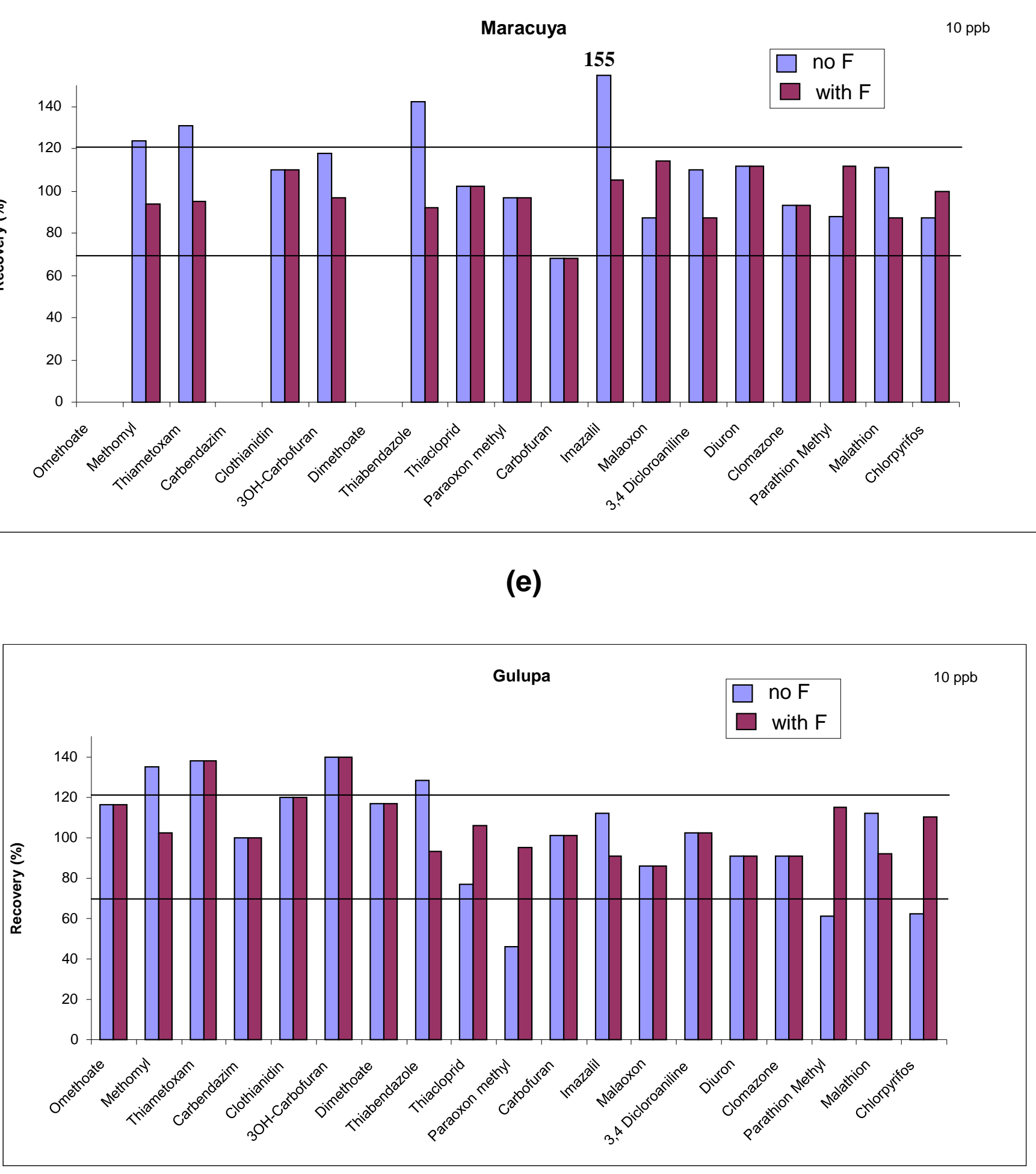


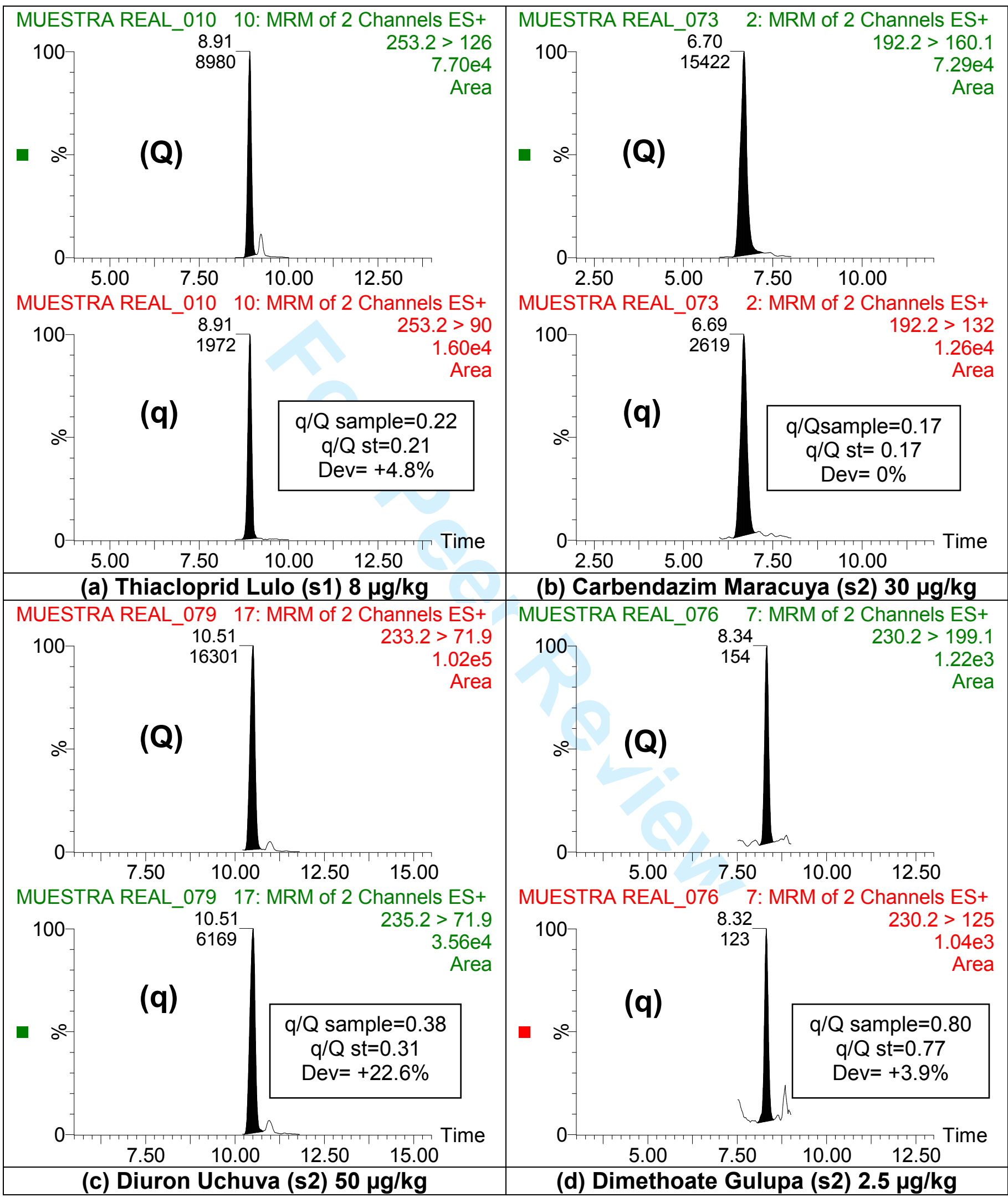

Figura 5 


\title{
SUPLEMENTARY INFORMATION
}

Exploring matrix effects in liquid chromatography-tandem mass spectrometry determination of pesticide residues in tropical fruits

\author{
A.M. Botero-Coy, J.M. Marín, R. Serrano, J.V. Sancho, F. Hernández* \\ Research Institute for Pesticides and Water, University Jaume I, 12071 Castellón, Spain \\ Tel 34-964-387366; Fax 34-964-387368; felix.hernandez@uji.es
}

In this section, three figures and two tables are included giving useful supplementary information for the readers. 
Table SI 1. Compounds and metabolites included in this work. Maximum Residue Limits (MRLs) as established in Regulation (EC) No 396/2005

\begin{tabular}{|c|c|c|c|c|c|c|c|c|c|c|c|c|c|c|}
\hline 1 Pesticide & Use & Metabolite & Granadilla & Maracuya & Gulupa & Mangostan & Tamarillo & Uchuva & Lulo & Carambolo & Feijoa & Guayaba & Pithaya & Papaya \\
\hline 1 4hethomyl & insecticide & & $0,02^{*}$ & $0,02^{*}$ & $0,02^{*}$ & $0,02^{*}$ & $0,02^{*}$ & $0,02^{*}$ & $0,02^{*}$ & $0,05^{*}$ & $0,05^{\star}$ & $0,05^{\star}$ & $0,05^{*}$ & $0,02^{*}$ \\
\hline Thiamethoxam & insecticide & clothianidin & $0,05^{*}$ & $0,05^{*}$ & $0,05^{*}$ & $0,05^{*}$ & 0,2 & 0,2 & $0,05^{\star}$ & $0,05^{*}$ & $0,05^{*}$ & $0,05^{*}$ & $0,05^{*}$ & $0,05^{*}$ \\
\hline $1 /\left.\right|_{5} ^{4}$ arbendazim & fungicide & & $0,1^{*}$ & $0,1^{*}$ & $0,1^{*}$ & $0,1^{*}$ & 0,3 & 0,3 & $0,1^{*}$ & $0,1^{*}$ & $0,1^{*}$ & $0,1^{*}$ & $0,1^{*}$ & 0,2 \\
\hline 1 Thiacloprid & insecticide & & $0,02^{*}$ & $0,02^{*}$ & $0,02^{*}$ & $0,02^{*}$ & 0,5 & 0,5 & $0,02^{*}$ & $0,02^{*}$ & $0,02^{*}$ & $0,02^{*}$ & $0,02^{*}$ & 0,5 \\
\hline 18 hiabendazole & fungicide & & $0,05^{*}$ & $0,05^{*}$ & $0,05^{*}$ & $0,05^{*}$ & $0,05^{*}$ & $0,05^{*}$ & $0,05^{*}$ & $0,05^{*}$ & $0,05^{*}$ & $0,05^{*}$ & $0,05^{*}$ & 10 \\
\hline 1 Carbofuran & insecticide & 3-OH-carbofuran & $0,01^{*}$ & $0,01^{*}$ & $0,01^{*}$ & $0,01^{*}$ & $0,01^{*}$ & $0,01^{*}$ & $0,01^{*}$ & $0,01^{*}$ & $0,01^{*}$ & $0,01^{*}$ & $0,01^{*}$ & $0,01^{*}$ \\
\hline Imazalil & fungicide & & $0,05^{*}$ & $0,05^{*}$ & $0,05^{*}$ & $0,05^{*}$ & 0,5 & 0,5 & $0,05^{\star}$ & $0,05^{*}$ & $0,05^{*}$ & $0,05^{*}$ & $0,05^{*}$ & $0,05^{*}$ \\
\hline 2 Picloram & herbicide & & $0,01^{*}$ & $0,01^{*}$ & $0,01^{*}$ & $0,01^{*}$ & $0,01^{*}$ & $0,01^{*}$ & $0,01^{*}$ & $0,01^{*}$ & $0,01^{*}$ & $0,01^{*}$ & $0,01^{*}$ & $0,01^{*}$ \\
\hline 23Diuron & herbicide & 3,4- dichloraniline & $0,01^{*}$ & $0,01^{*}$ & $0,01^{*}$ & $0,01^{*}$ & $0,01^{*}$ & $0,01^{*}$ & $0,01^{*}$ & $0,01^{*}$ & $0,01^{*}$ & $0,01^{*}$ & $0,01^{*}$ & $0,01^{*}$ \\
\hline 24 Clomazone & herbicide & & $0,01^{*}$ & $0,01^{*}$ & $0,01^{*}$ & $0,01^{*}$ & $0,01^{*}$ & $0,01^{*}$ & $0,01^{*}$ & $0,01^{*}$ & $0,01^{*}$ & $0,01^{*}$ & $0,01^{*}$ & $0,01^{*}$ \\
\hline 25 Malathion & insecticide-acaricide & malaoxon & $0,02^{*}$ & $0,02^{*}$ & $0,02^{*}$ & $0,02^{*}$ & $0,02^{*}$ & $0,02^{*}$ & $0,02^{*}$ & $0,02^{*}$ & $0,02^{*}$ & $0,02^{*}$ & $0,02^{*}$ & $0,02^{*}$ \\
\hline 20 & insecticide, acaricide & paraoxon-methyl & $0,01^{*}$ & $0,01^{*}$ & $0,01^{*}$ & $0,01^{*}$ & $0,01^{*}$ & $0,01^{*}$ & $0,01^{*}$ & $0,01^{*}$ & $0,01^{*}$ & $0,01^{*}$ & $0,01^{*}$ & $0,01^{*}$ \\
\hline 28Chlorpyrifos & insecticide & & $0,05^{*}$ & $0,05^{*}$ & $0,05^{*}$ & $0,05^{*}$ & 0,5 & 0,5 & $0,05^{*}$ & $0,05^{*}$ & $0,05^{*}$ & $0,05^{*}$ & $0,05^{*}$ & $0,05^{*}$ \\
\hline
\end{tabular}

*Limit of determination of the analytical method

Bold number indicate that GLP residue trials have been performed in order to set up the MRL 
Table SI 2a. Recoveries (\%) obtained for the six matrices subjected to validation using concentrations calculated with direct calibration (direct) and corrected after application of the matrix effect factors (corrected). Fortification level $0.01 \mathrm{mg} / \mathrm{kg}(\mathrm{n}=5)$

\begin{tabular}{|c|c|c|c|c|c|c|c|c|c|c|c|c|}
\hline \multirow[t]{2}{*}{ Matrix } & \multicolumn{2}{|c|}{ Granadilla } & \multicolumn{2}{|c|}{ Tamarillo } & \multicolumn{2}{|c|}{ Uchuva } & \multicolumn{2}{|c|}{ Pithaya } & \multicolumn{2}{|c|}{ Maracuya } & \multicolumn{2}{|c|}{ Gulupa } \\
\hline & direct & corrected & direct & corrected & direct & corrected & direct & corrected & direct & corrected & direct & corrected \\
\hline Pesticide & & & & & & & & & & & & \\
\hline Omethoate & 92 & 92 & 81 & 81 & 104 & 104 & 86 & 86 & * & * & 116 & 116 \\
\hline Methomyl & 97 & 70 & 100 & 78 & 135 & 107 & 103 & 103 & 124 & 94 & 135 & 102 \\
\hline Thiametoxan & 123 & 91 & 123 & 96 & 176 & 114 & 130 & 92 & 131 & 95 & 138 & 138 \\
\hline Carbendazim & $*$ & $*$ & $*$ & $*$ & 114 & 114 & 108 & 108 & $*$ & $*$ & 100 & 100 \\
\hline Clothianidin & 63 & 91 & 105 & 105 & 126 & 126 & 100 & 100 & 110 & 110 & 120 & 120 \\
\hline 3OH-Carbofuran & 115 & 115 & 109 & 86 & 159 & 120 & 112 & 112 & 118 & 97 & 140 & 140 \\
\hline Dimethoate & 87 & 87 & 94 & 94 & 135 & 135 & 106 & 106 & $*$ & $*$ & 117 & 117 \\
\hline Thiabendazole & 206 & 84 & 210 & 84 & 196 & 80 & 276 & 99 & 142 & 92 & 128 & 93 \\
\hline Thiacloprid & 50 & 75 & 97 & 97 & 91 & 91 & 92 & 92 & 102 & 102 & 77 & 106 \\
\hline Paraoxon methyl & 54 & 86 & 88 & 88 & 89 & 89 & 78 & 105 & 97 & 97 & 46 & 95 \\
\hline Carbofuran & 70 & 70 & 70 & 70 & 69 & 99 & 58 & 58 & 68 & 68 & 101 & 101 \\
\hline Imazalil & 113 & 74 & 116 & 75 & 129 & 84 & 128 & 88 & 155 & 105 & 112 & 91 \\
\hline Malaoxon & 78 & 78 & 46 & 46 & 81 & 102 & 70 & 70 & 87 & 114 & 86 & 86 \\
\hline 3,4 Dicloroaniline & 73 & 52 & 53 & 43 & 65 & 65 & 61 & 47 & 110 & 87 & 102 & 102 \\
\hline Diuron & 103 & 103 & 89 & 89 & 109 & 109 & 95 & 95 & 112 & 112 & 91 & 91 \\
\hline Clomazone & 84 & 84 & 78 & 78 & 52 & 103 & 81 & 81 & 93 & 93 & 91 & 91 \\
\hline Parathion Methyl & 62 & 103 & 64 & 87 & 68 & 103 & 80 & 106 & 88 & 112 & 61 & 115 \\
\hline Malathion & 89 & 72 & 73 & 73 & 118 & 118 & 88 & 88 & 111 & 87 & 112 & 92 \\
\hline Chlorpyrifos & 112 & 112 & 64 & 90 & 29 & 106 & 67 & 98 & 87 & 100 & 62 & 110 \\
\hline
\end{tabular}

* Data not available due to the presence of the analyte in the sample used for validation 
Table SI 2b. Recoveries (\%) obtained for the six matrices subjected to validation using concentrations calculated with direct calibration (direct) and corrected after application of the matrix effect factors (corrected). Fortification level $0.1 \mathrm{mg} / \mathrm{kg}(\mathrm{n}=5)$

\begin{tabular}{|c|c|c|c|c|c|c|c|c|c|c|c|c|}
\hline \multirow[t]{2}{*}{ Matrix } & \multicolumn{2}{|c|}{ Granadilla } & \multicolumn{2}{|c|}{ Tamarillo } & \multicolumn{2}{|c|}{ Uchuva } & \multicolumn{2}{|c|}{ Pithaya } & \multicolumn{2}{|c|}{ Maracuya } & \multicolumn{2}{|c|}{ Gulupa } \\
\hline & direct & corrected & direct & corrected & direct & corrected & direct & corrected & direct & corrected & direct & corrected \\
\hline Pesticide & & & & & & & & & & & & \\
\hline Omethoate & 103 & 103 & 84 & 84 & 108 & 108 & 85 & 85 & 84 & 84 & 117 & 117 \\
\hline Methomyl & 106 & 76 & 103 & 80 & 136 & 109 & 104 & 104 & 127 & 97 & 129 & 98 \\
\hline Thiametoxan & 137 & 101 & 108 & 84 & 161 & 104 & 122 & 86 & 136 & 99 & 104 & 104 \\
\hline Carbendazim & $*$ & $*$ & 50 & 50 & 107 & 107 & 105 & 105 & 86 & 110 & 108 & 108 \\
\hline Clothianidin & 70 & 102 & 96 & 96 & 127 & 127 & 94 & 94 & 118 & 97 & 114 & 114 \\
\hline 3OH-Carbofuran & 122 & 122 & 98 & 78 & 159 & 120 & 116 & 116 & 126 & 104 & 124 & 124 \\
\hline Dimethoate & 92 & 92 & 88 & 88 & 131 & 131 & 104 & 104 & 97 & 97 & 110 & 110 \\
\hline Thiabendazole & 204 & 83 & 184 & 74 & 187 & 76 & 242 & 87 & 125 & 81 & 57 & 71 \\
\hline Thiacloprid & 53 & 79 & 88 & 88 & 87 & 87 & 92 & 92 & 95 & 95 & 69 & 95 \\
\hline Paraoxon methyl & 55 & 88 & 77 & 77 & 84 & 84 & 76 & 102 & 93 & 93 & 44 & 94 \\
\hline Carbofuran & 81 & 81 & 79 & 79 & 85 & 120 & 60 & 60 & 84 & 84 & 106 & 106 \\
\hline Imazalil & 113 & 74 & 111 & 72 & 130 & 84 & 110 & 76 & 117 & 80 & 106 & 86 \\
\hline Malaoxon & 86 & 86 & 55 & 55 & 98 & 124 & 77 & 77 & 96 & 127 & 92 & 92 \\
\hline Diuron & 104 & 104 & 84 & 84 & 107 & 107 & 92 & 92 & 110 & 110 & 87 & 87 \\
\hline Clomazone & 88 & 88 & 77 & 77 & 52 & 104 & 81 & 81 & 95 & 95 & 87 & 87 \\
\hline Parathion Methyl & 58 & 94 & 57 & 77 & 60 & 90 & 64 & 85 & 92 & 117 & 63 & 119 \\
\hline Malathion & 103 & 83 & 79 & 79 & 127 & 127 & 96 & 96 & 118 & 92 & 115 & 94 \\
\hline Chlorpyrifos & 73 & 73 & 59 & 81 & 28 & 102 & 55 & 92 & 76 & 100 & 64 & 114 \\
\hline
\end{tabular}

${ }^{*}$ Data not available due to the presence of the analyte in the sample used for validation 
Figure SI 1. QuEChERS procedure applied in this work

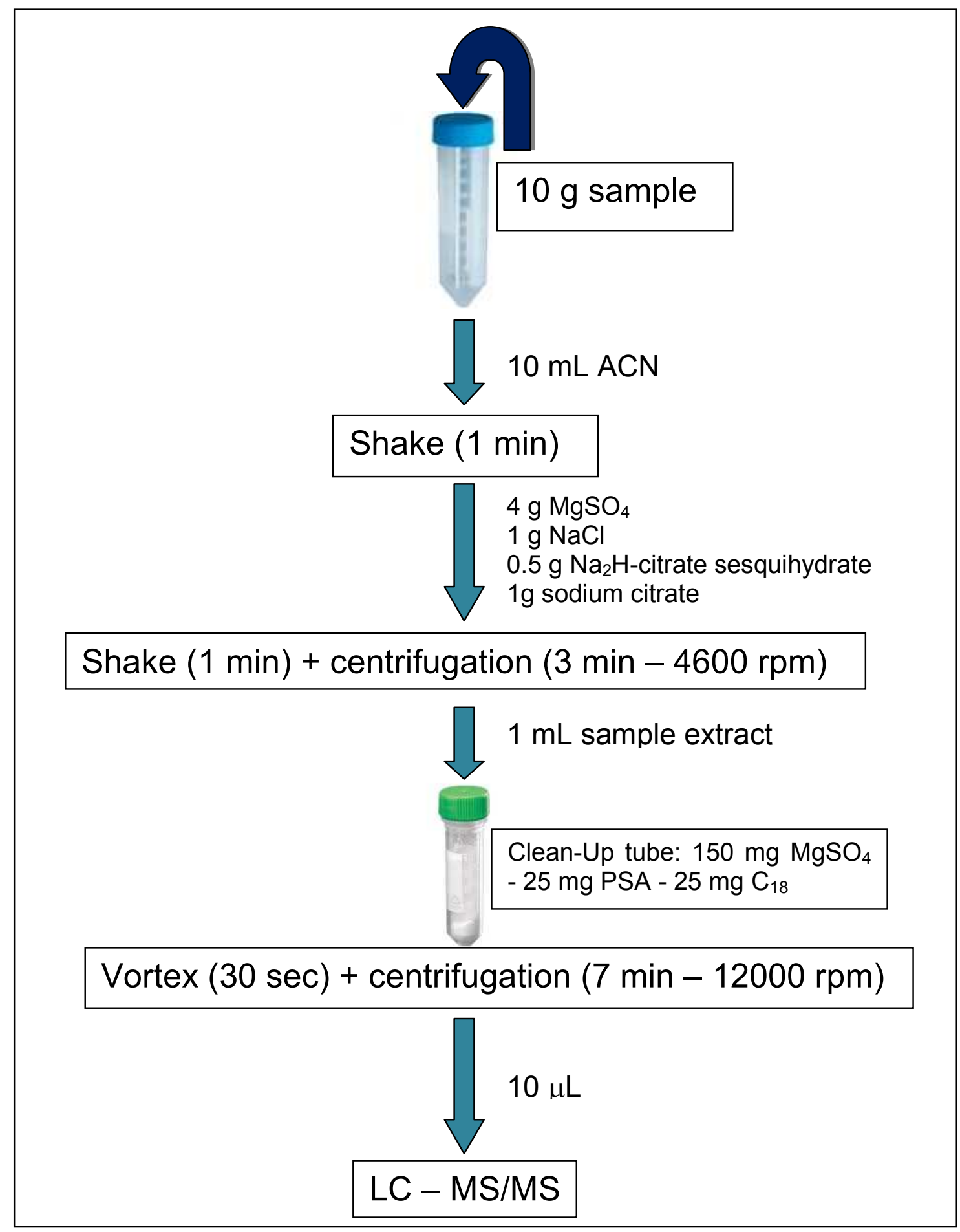


Figure SI 2. Correction factor calculation

$$
\begin{aligned}
& \frac{\text { Area }}{\text { Direct Slope }} \times F=\frac{\text { Area }}{\text { Direct Slope } 1(\% \text { Difference } \times \text { Direct Slops }) / 100)} \\
& \frac{F}{\text { Direct Slope }}=\frac{1}{\text { Direct Slope }+((\% \text { Difference } \times \text { Direct Slope }) / 100)} \\
& F=\frac{\text { Direcl slope }}{\text { Direct Slcpe }+((\% \text { Difference } \times \text { Direct Slope }) / 100)} \\
& F=\frac{\text { Direct Slope }}{\frac{100 \times \text { Direct Slope }}{100}+\frac{(\% \text { Difference } \times \text { Direct Slope }}{100}} \\
& F=\frac{1}{1+\frac{\% \text { Difjerence }}{100}}
\end{aligned}
$$


Figure SI 3. Calibration graphs obtained for selected pesticides in different sample matrices. Calibration in solvent and $\pm 20 \%$ tolerance in the slope is highlighted in yellow
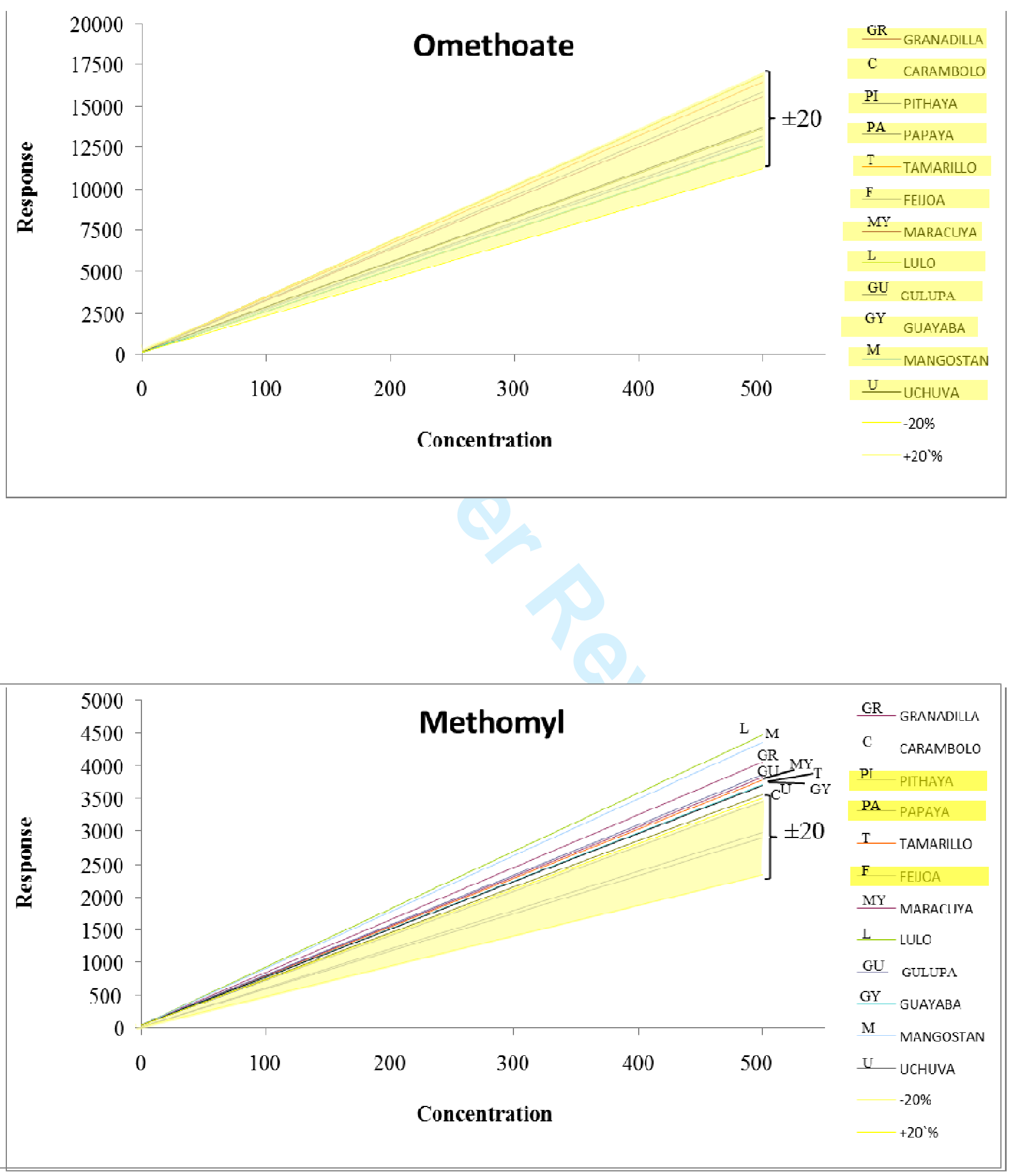

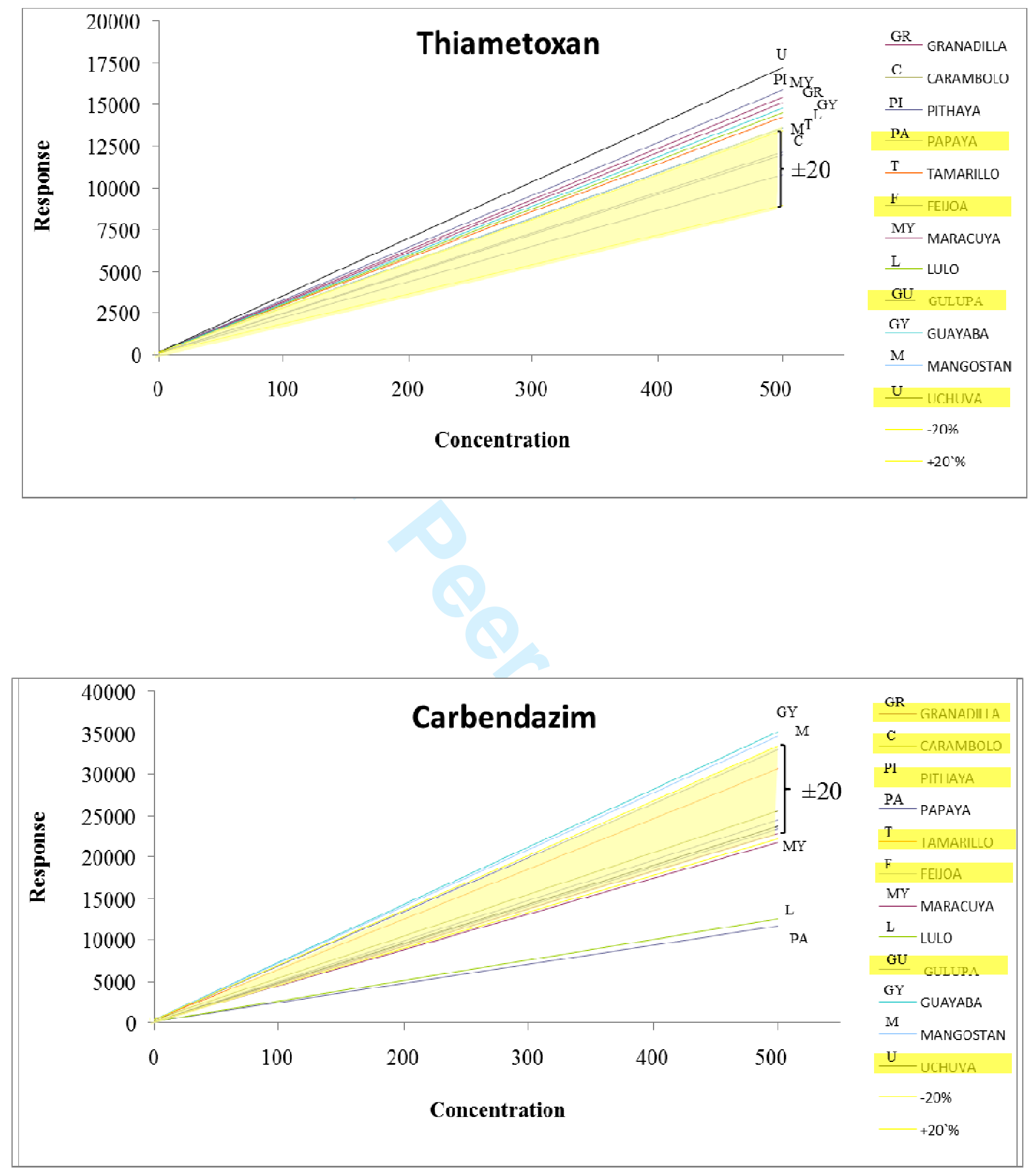

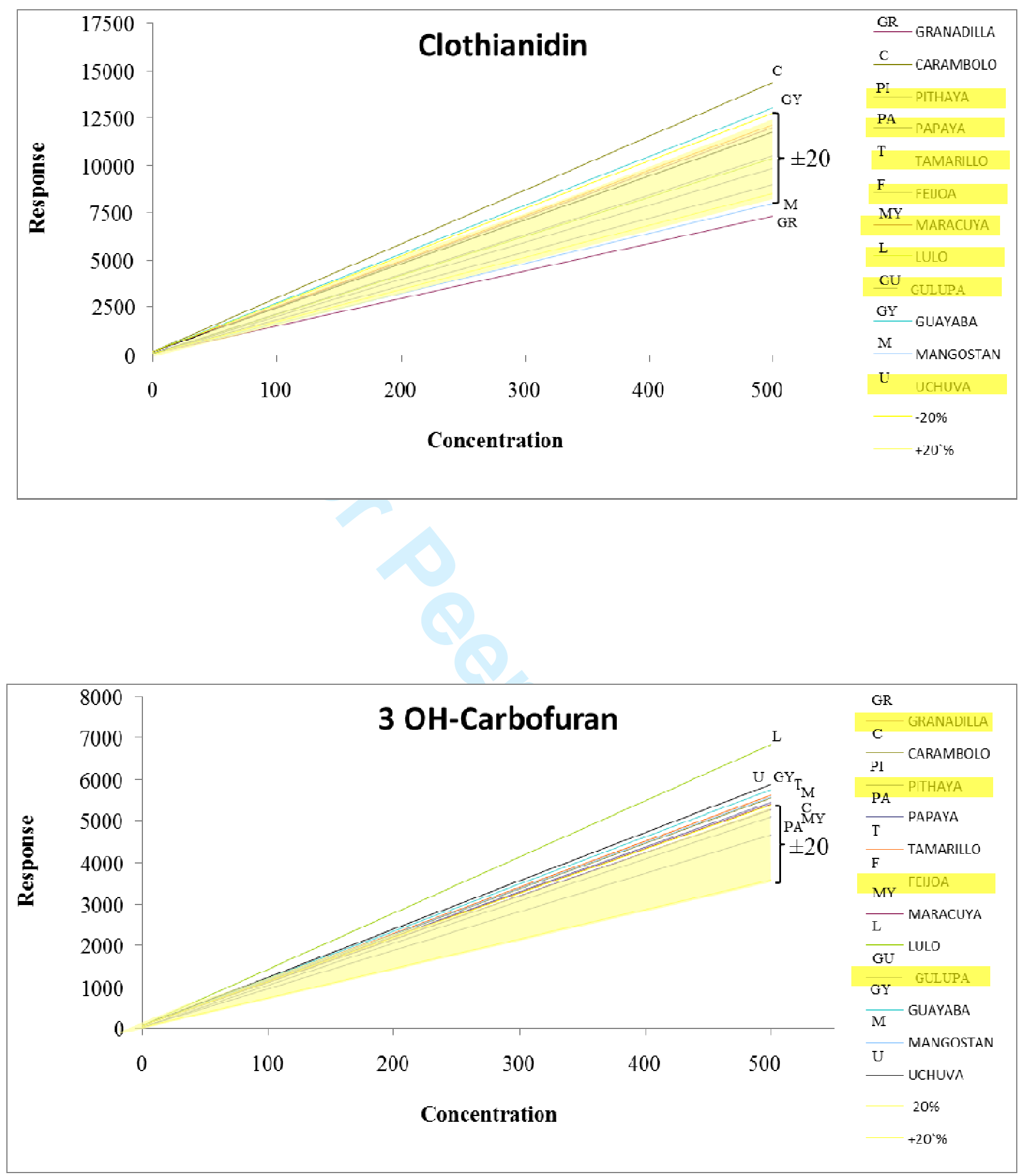

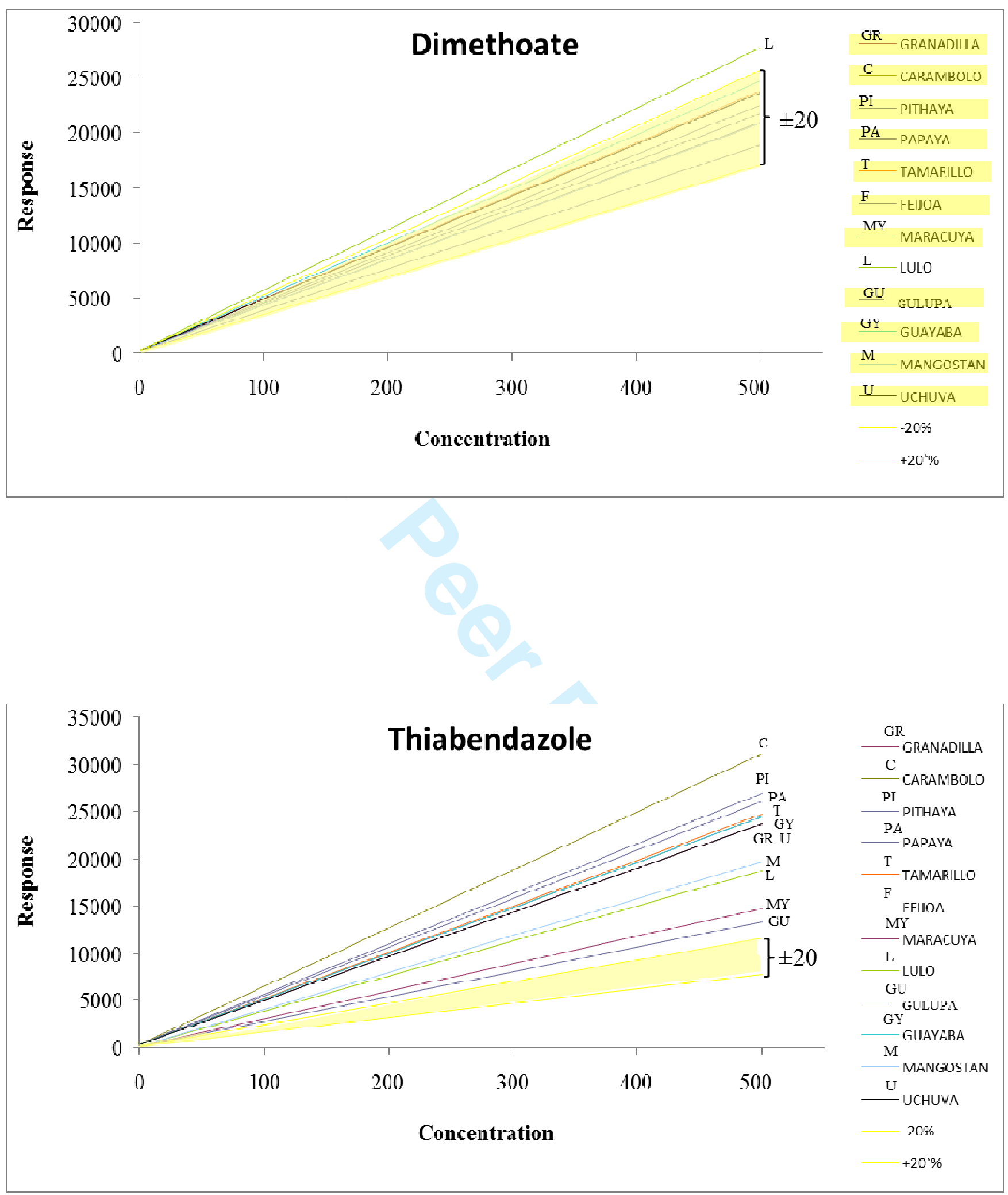

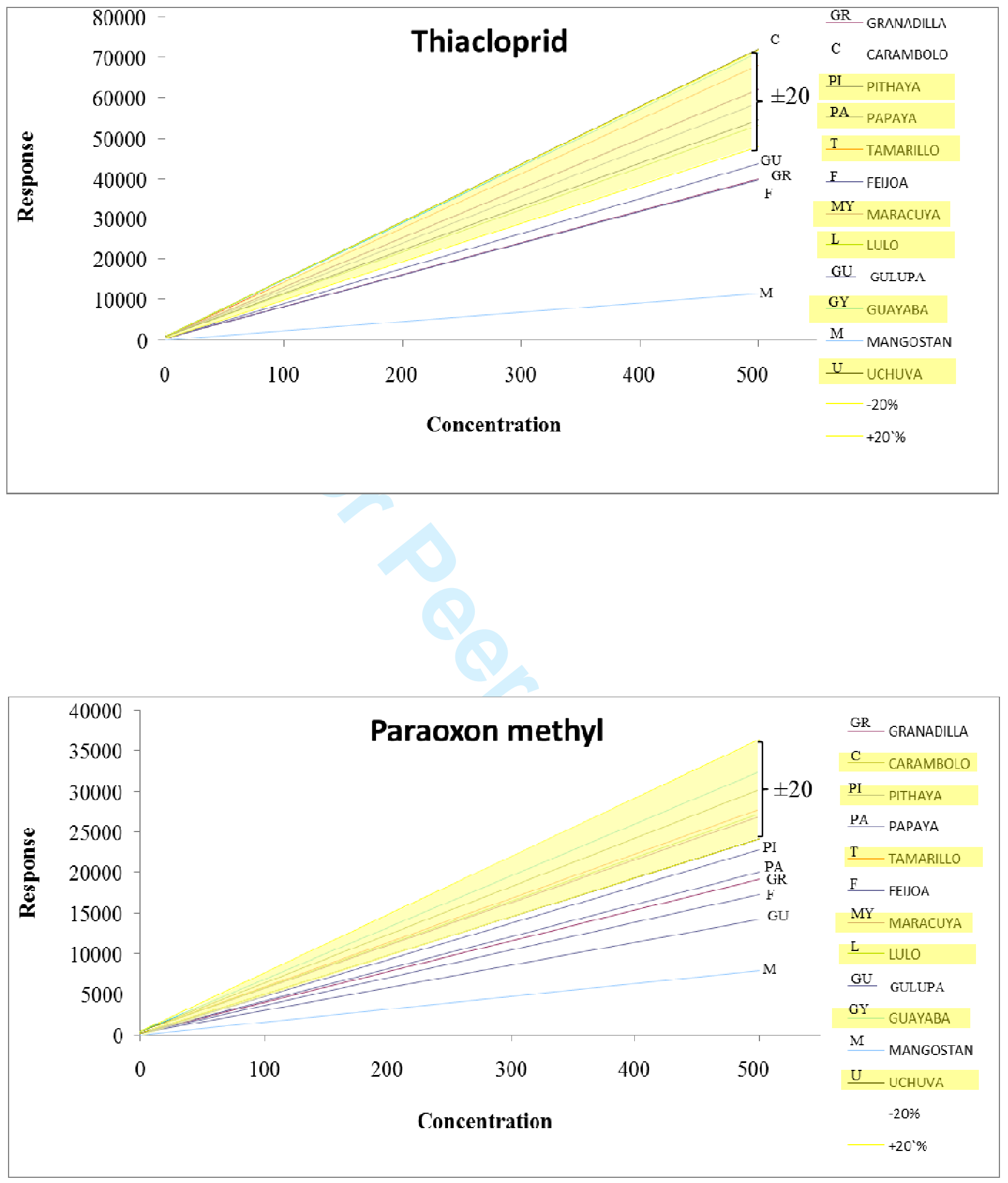

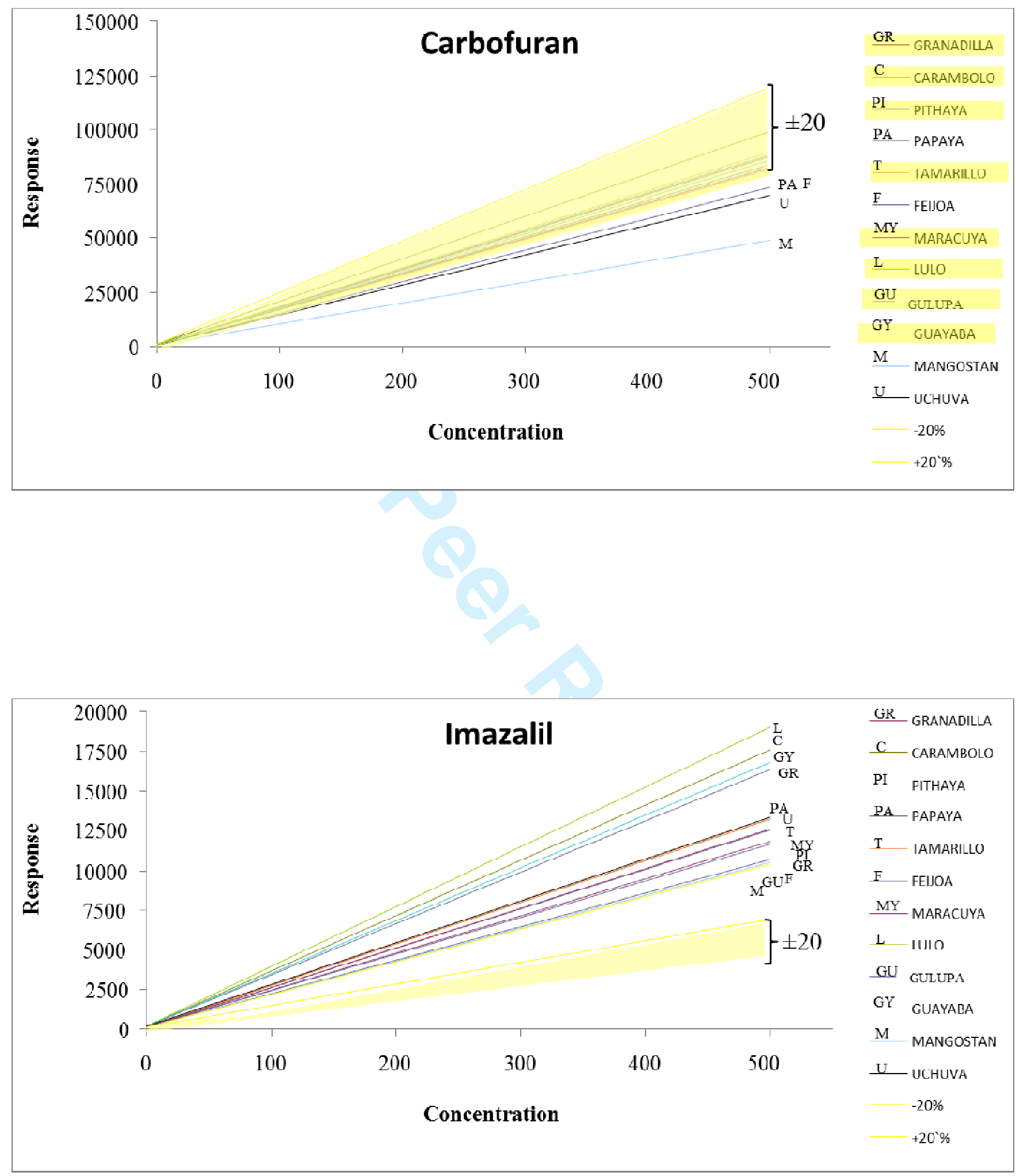

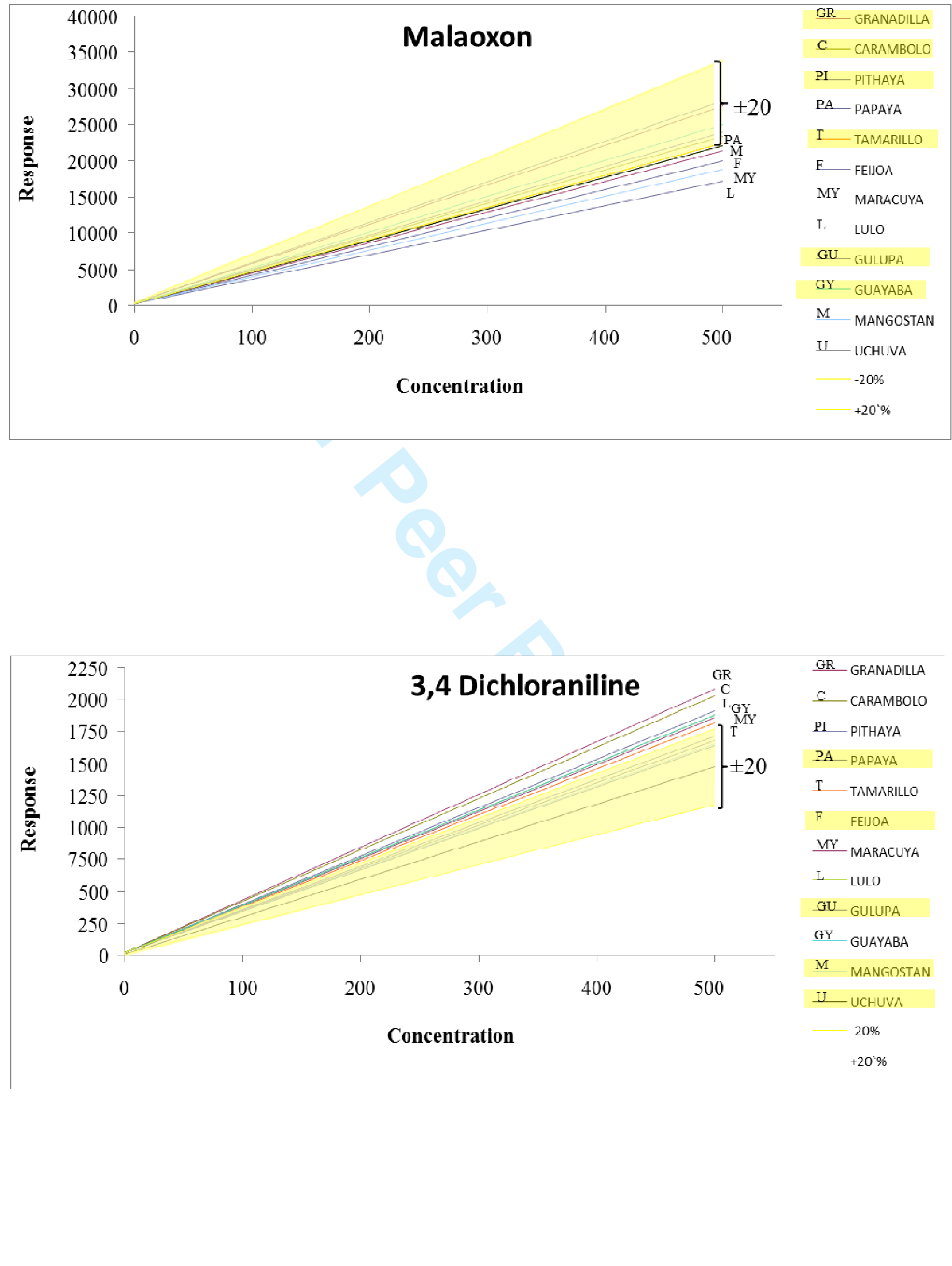

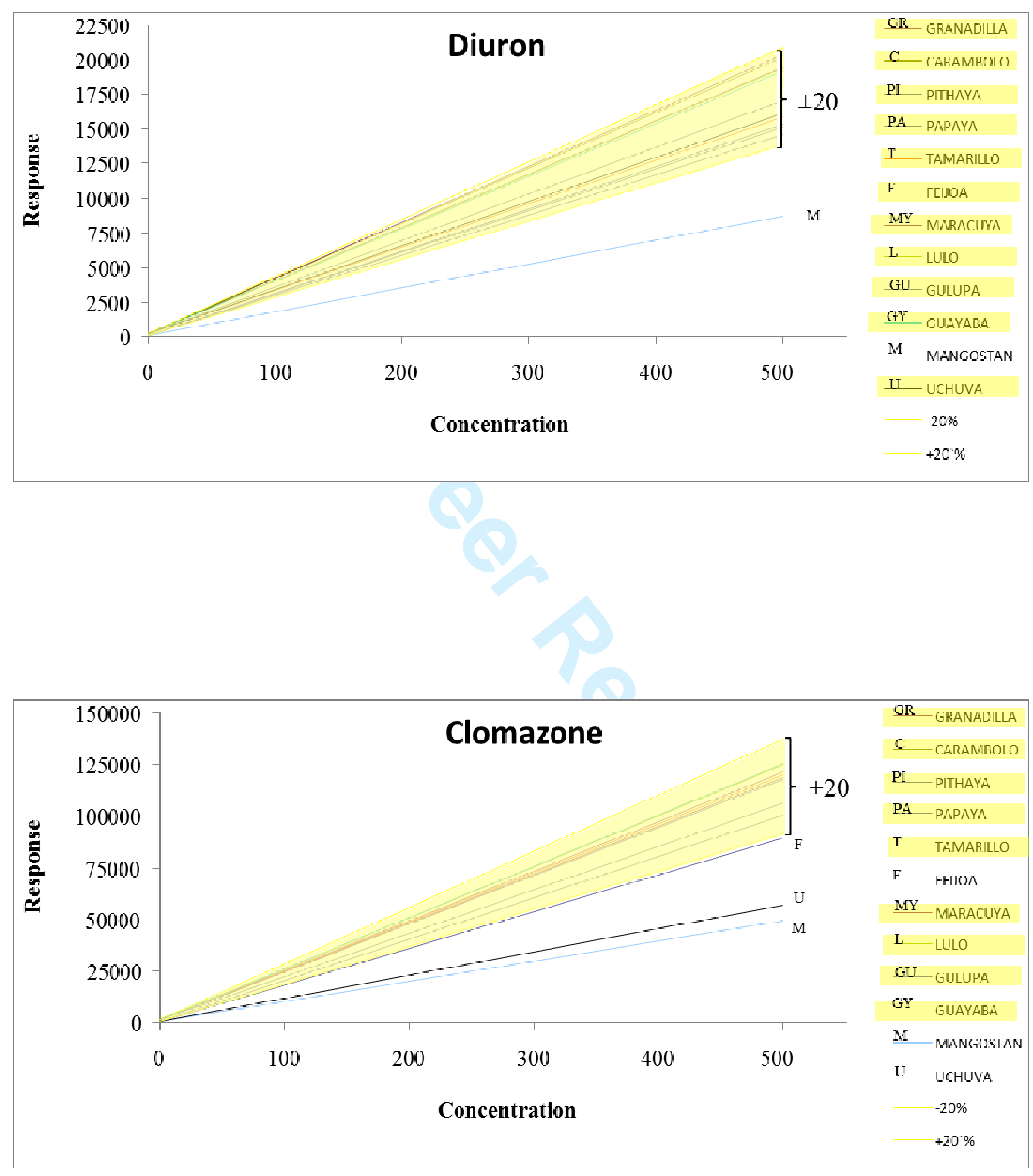

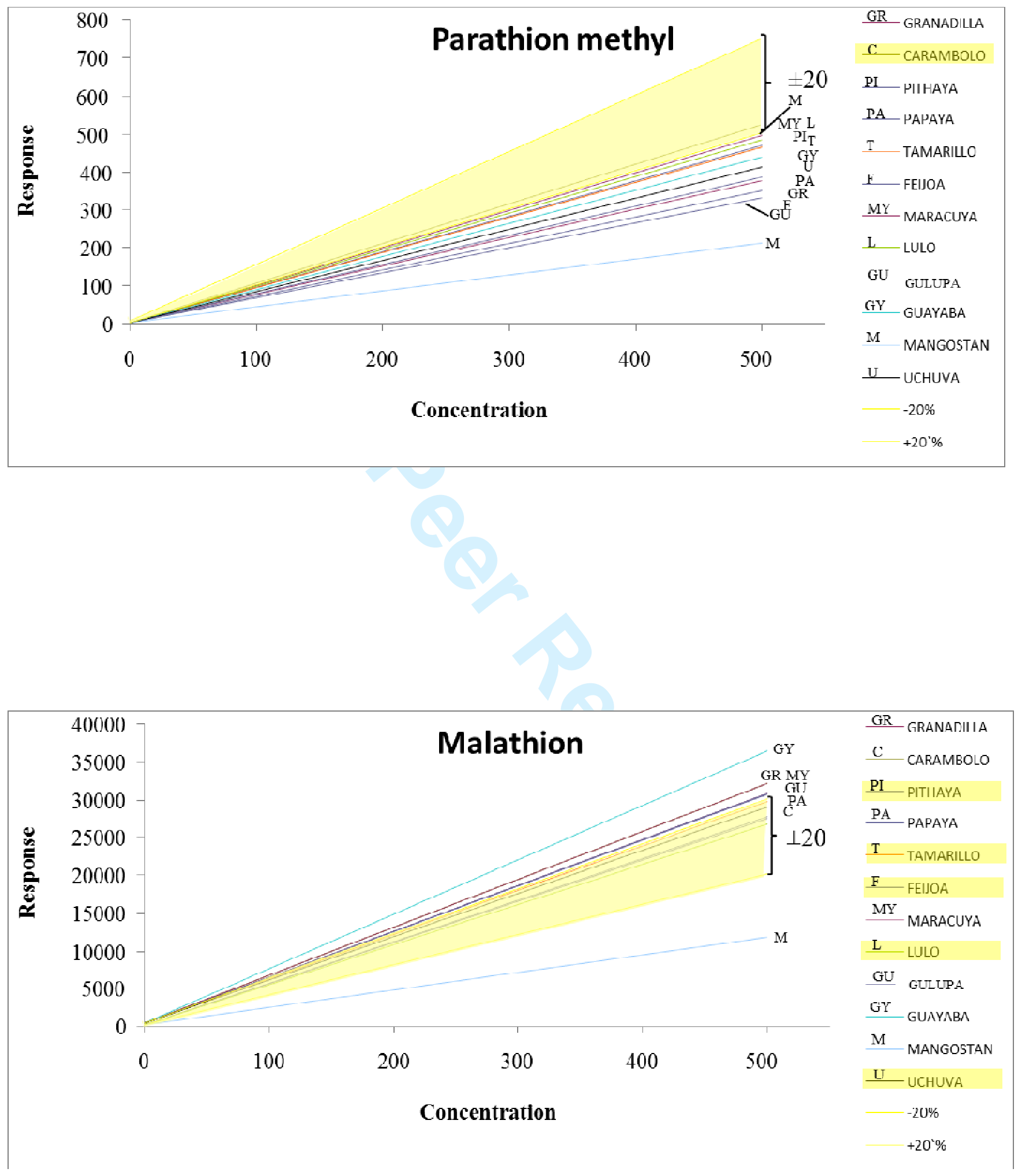


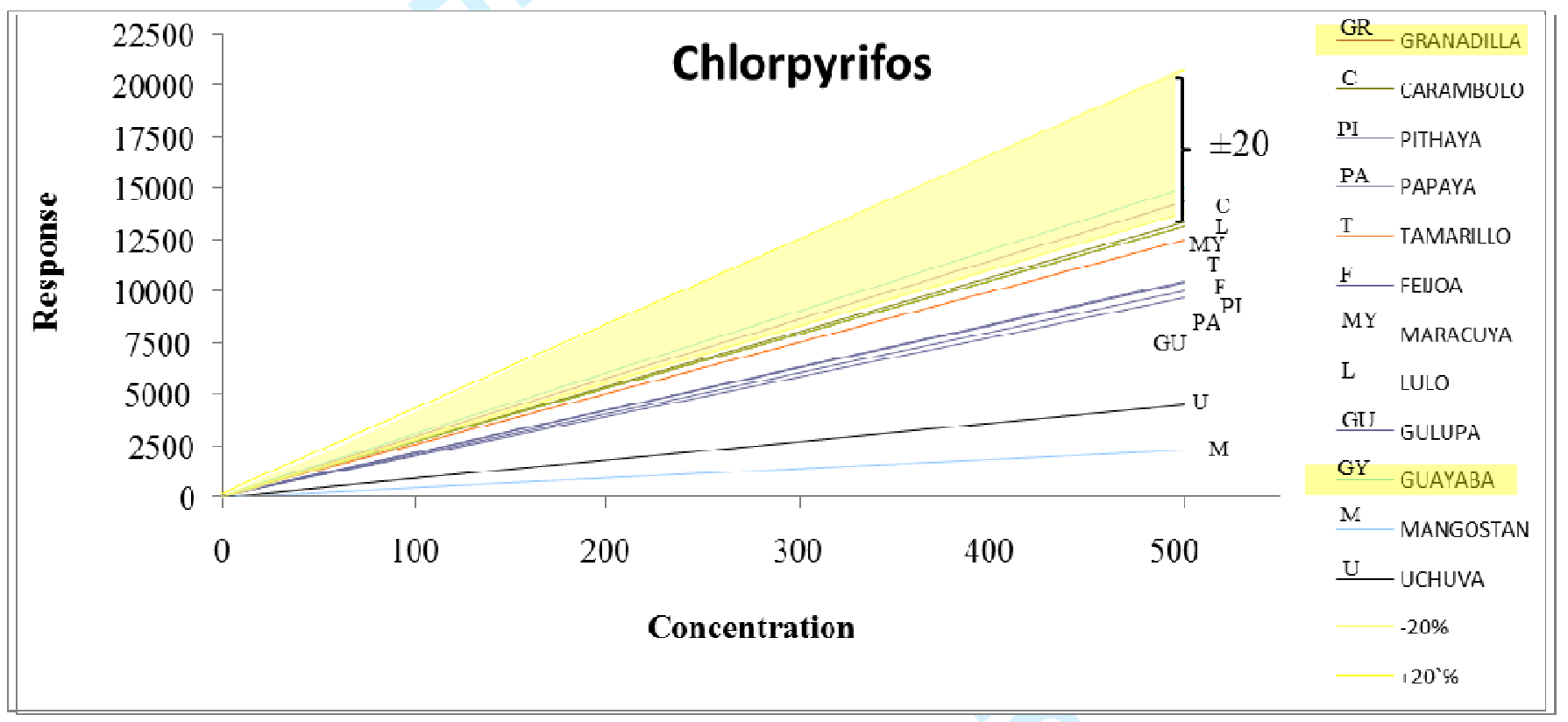

